\title{
Direct Observation of Intermediates from the Ring-opening of Cetylpyridinium Chloride by Surface-enhanced Raman Spectroscopy
}

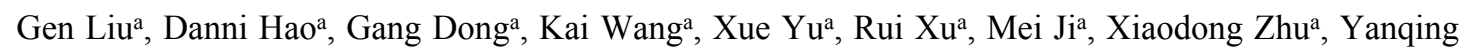
$\mathrm{Ma}^{\mathrm{a}, \mathrm{b}^{*}}$, Lei $\mathrm{Ma}^{\mathrm{a}}$

a Tianjin International Center for Nanoparticles and Nanosystems, Tianjin University, 300072 , P.R.China

b State Laboratory of Precision Measuring Technology and Instruments, Tianjin University, Tianjin 300072, China

Corresponding author: E-mail: mayanqing@tju.edu.cn; E-mail: lei.ma@tju.edu.cn

\section{Experimental}

Reagents. Gold (III) chloride trihydrate $\left(\mathrm{HAuCl}_{4} \cdot 3 \mathrm{H}_{2} \mathrm{O}, 99.9+\%\right)$, sodium borohydrate $\left(\mathrm{NaBH}_{4}, 99.99 \%\right.$ ), L-ascorbic acid (AA, 99+\%), potassium bromide $(\mathrm{KBr})$, cetyltrimethylammonium bromide (CTAB, 99.9\%) and cetylpyridinium chloride (CPC, 99\%) were purchased from Aladdin, and used for syntheses without further purification. Milli-Q ultrapure deionized water (18.2 M $\Omega$, Millipore UV Plus) was used in preparing all the aqueous solutions.

\subsection{Synthesis of Au NPs ${ }^{1}$}

Synthesis of Au seeds refers to the reported method. The Au nano-octahedral seeds were prepared using a two-step procedure. Firstly, $3 \mathrm{~nm}$ Au seeds were prepared by quickly injecting $0.60 \mathrm{~mL}$ of ice-cold, freshly prepared $\mathrm{NaBH}_{4}(10 \mathrm{mM})$ into a rapidly stirred mixture of $\mathrm{HAuCl}_{4}(10 \mathrm{mM}, 0.25 \mathrm{~mL})$ and CTAB $(0.1 \mathrm{M}, 9.75 \mathrm{~mL})$. The seed solution was stirred for 2 minutes and then left undisturbed at $25{ }^{\circ} \mathrm{C}$ for 3 hours to 
ensure complete decomposition of remaining $\mathrm{NaBH}_{4}$ in the solution. The seed solution was diluted 100 times with $0.1 \mathrm{M} \mathrm{CTAB}$. For synthesizing octahedral Au seeds, $4 \mathrm{~mL}$ of aqueous $\mathrm{HAuCl}_{4}$ solution $(0.3 \mathrm{mM}), 24 \mathrm{~mL}$ of aqueous CTAB solution $(20 \mathrm{mM})$, and $1.8 \mathrm{~mL}$ of aqueous AA solution $(100 \mathrm{mM})$ were mixed, followed by adding $360 \mu \mathrm{L}$ of the diluted seed solution while stirring. The solution mixture was then left to age at $25^{\circ} \mathrm{C}$ for 12 hours. The resulting $\mathrm{Au}$ nano-octahedral seeds were washed 3 times with $0.1 \mathrm{M} \mathrm{CPC}$ solution by centrifugation $(12000 \mathrm{rpm}, 10 \mathrm{~min})$ and finally condensed in $3 \mathrm{~mL}$ of $0.1 \mathrm{M}$ CPC solution. Synthesis of $\sim 45 \mathbf{~ n m ~ A u}$ nanoparticles (Au NPs): $500 \mu \mathrm{L}$ of $100 \mathrm{mM} \mathrm{KBr}$ solution, $100 \mu \mathrm{L}$ of $10 \mathrm{mM}$ $\mathrm{HAuCl}_{4}$ solution, $150 \mu \mathrm{L}$ of freshly prepared $100 \mathrm{mM} \mathrm{AA}$, and $270 \mu \mathrm{L}$ of the synthesized $\mathrm{Au}$ seed solution were added to $5 \mathrm{~mL}$ of $0.1 \mathrm{M} \mathrm{CPC}$ solution at $32{ }^{\circ} \mathrm{C}$ consecutively. The reaction was left undisturbed for $6 \mathrm{~h}$ and stopped by centrifugation (10000 rpm, $10 \mathrm{~min}$ ). The nanocrystals solution obtained was washed twice with Milli-Q water and concentrated for further characterizations and measurements.

\subsection{Fabrication of the self-assembled Au SERS platform ${ }^{2}$}

Si wafer was ultrasonic cleaned by acetone, ethanol and Milli-Q water for $30 \mathrm{~min}$, respectively. Then the wafer was dried by $\mathrm{N}_{2} .10 .0 \mu \mathrm{L}$ of fresh prepared Au NPs suspension was dropped on a cleaned Si substrate using a pipette. The droplet was allowed to evaporate on the substrate in a sealed glass jar containing saturation $\mathrm{K}_{2} \mathrm{SO}_{4}$ solution. The relativity humidity $(\mathrm{RH})$ in the jar was maintained at $96 \%$. Then the sealed glass jar was placed into an incubator with temperature set to $30{ }^{\circ} \mathrm{C}$, as shown in Figure S1. The evaporation process lasted for more than $12 \mathrm{~h}$. To exclude the interference of intrinsic peaks of Si substrate, and to position and characterize easily. The SERS platforms were prepared using a lithographic approach. The preparation of the patterned substrate, a $4400 \times 4400 \mu \mathrm{m}^{2}$ area with the minimum lithographic size $5 \mu \mathrm{m}$ was lithographed to get dot matrix on the cleaned silicon wafer surface using laser lithography system (Microlab 4A-100, SVG Optronics), followed by evaporating $5 \mathrm{~nm} \mathrm{Cr}$ and $35 \mathrm{~nm} \mathrm{Au} \mathrm{using} \mathrm{electron} \mathrm{beam} \mathrm{evaporation} \mathrm{(DZS500,} \mathrm{Shenyang}$ Scientific Instrument Co., Ltd), followed by a lift-off process to get the Au film arrays. The Au NPs assembly was performed using the method described above. The assembled samples were completely dried in air, then soaked in $50 \mathrm{~mL}$ deionized 
water for $3 \mathrm{~h}$, the soaked process was repeated 3 times, to completely remove the free-molecular. The obtained samples are dried in air for further characterizations and Raman measurements.

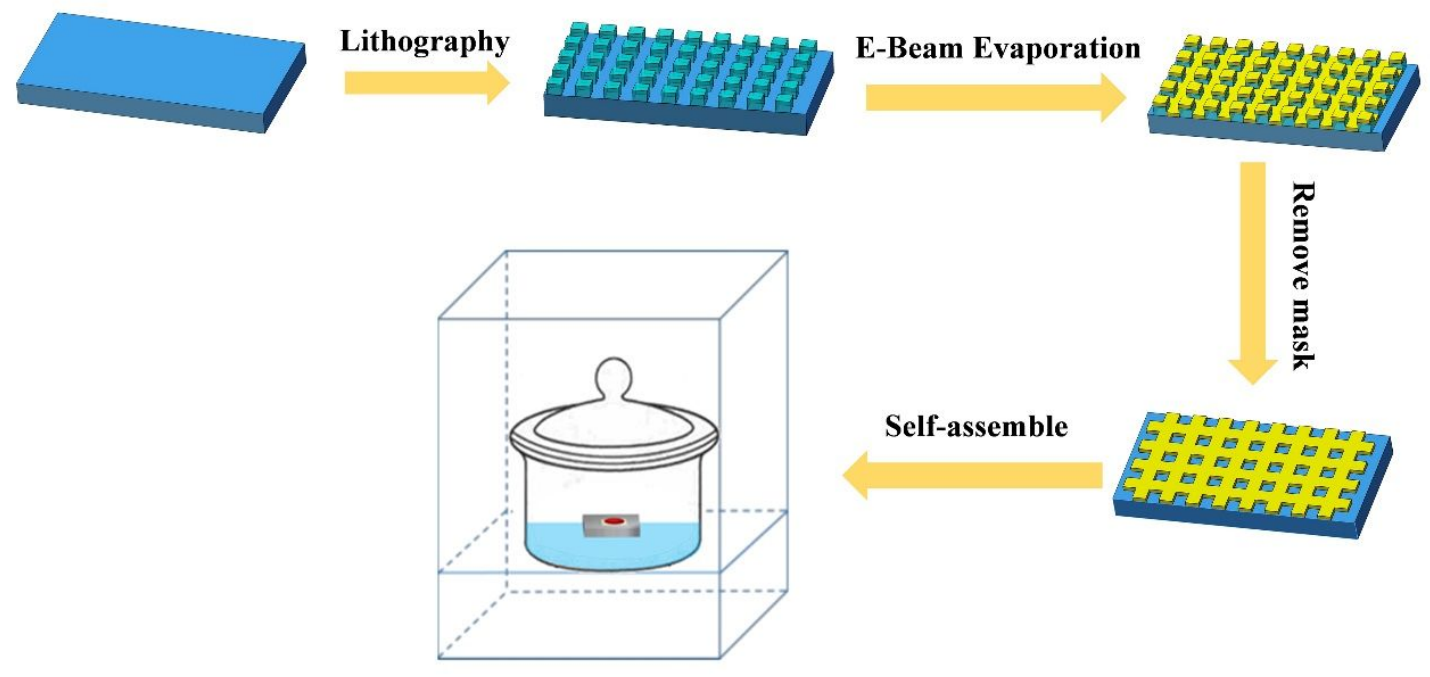

Figure S1. The schematic of SERS platform self-assembled.

\subsection{Characterization}

The Au NPs were characterized by SEM (SU3500, Hitachi, Japan), Energy Dispersive Spectrometer (EDS) (Model 550i, IXRF, America) and AFM (Park X-10) for its morphology and geometry. The information of the molecular structure and valence state of the sample was characterized by X-ray optical electron spectroscopy XPS (PHI 1600, ESCA system).The optical extinction spectrum of the sample is measured for nanoparticles self-assembled on the ITO glass, using the ultraviolet-visible spectrophotometer(UV-Vis, UV-3900, Hitachi, Japan). The SERS measurements were carried out on the Raman spectrograph (RTS-2, Titan Electro-Optics (Hong Kong) Co. Ltd). $532 \mathrm{~nm}$ and $785 \mathrm{~nm}$ laser were used to record the change of the sample Raman signal in dynamic mode. A home-made sample cell was used to carry out the SERS measurement in the aqueous, $\mathrm{N}_{2}$ and Ar gas atmosphere.

1.4 FDTD calculation: The classical finite difference time domain electrodynamics simulations were employed to compute the local near field intensities of Au systems that modelled our SERS platform, as shown in Figure S2. In the FDTD studies, the used model consisting of two nanospheres $(\mathrm{d}=45 \mathrm{~nm})$ and $\mathrm{Au}$ film positioned at different configurations. The Gap X and Gap Z were set at 1、2、3、4 and $5 \mathrm{~nm}$. 
The incident laser source direction is along the Z-axis, which is in accordance with the SERS experiments.

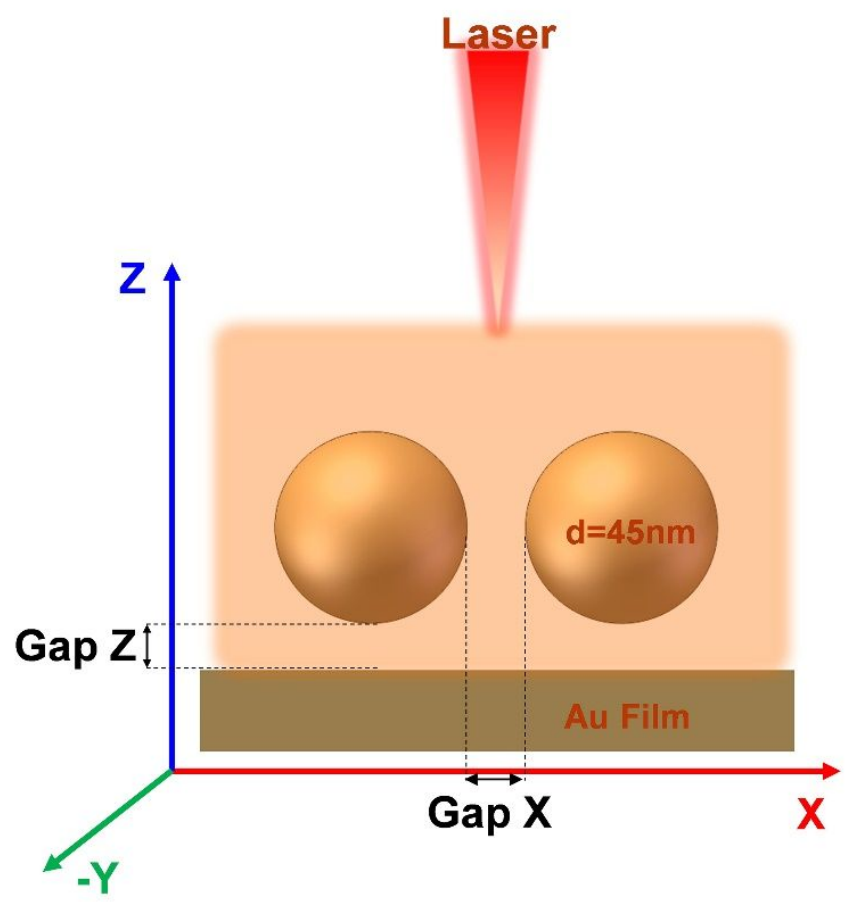

Figure S2. The schematic of FDTD simulations.

\subsection{DFT calculation}

All density functional theory calculations were performed by Gaussian 09 package. ${ }^{3}$ The hybrid exchange-correlation functional B3LYP, the basis sets for $\mathrm{H}, \mathrm{C}$, $\mathrm{N}$ atoms of the investigated molecules were $6-311+\mathrm{g}(\mathrm{d}, \mathrm{p})$, and all gold atoms were used by LANL2DZ basis sets. The graphs of the clusters were rendered by Visual Molecular Dynamics (VMD) software. ${ }^{4-6}$ The structure and name of the products refer to the reference. ${ }^{6}$ The simulated Raman spectra were presented in terms of a Lorentzian expansion with a line width $40 \mathrm{~cm}^{-1}$ and excitation wavelength of $785 \mathrm{~nm}$. The calculated harmonic frequencies were scaled by the factor of $0.981(0.967)$ for below $2000 \mathrm{~cm}^{-1}$ (above $\left.2000 \mathrm{~cm}^{-1}\right) .5,7,8$ 

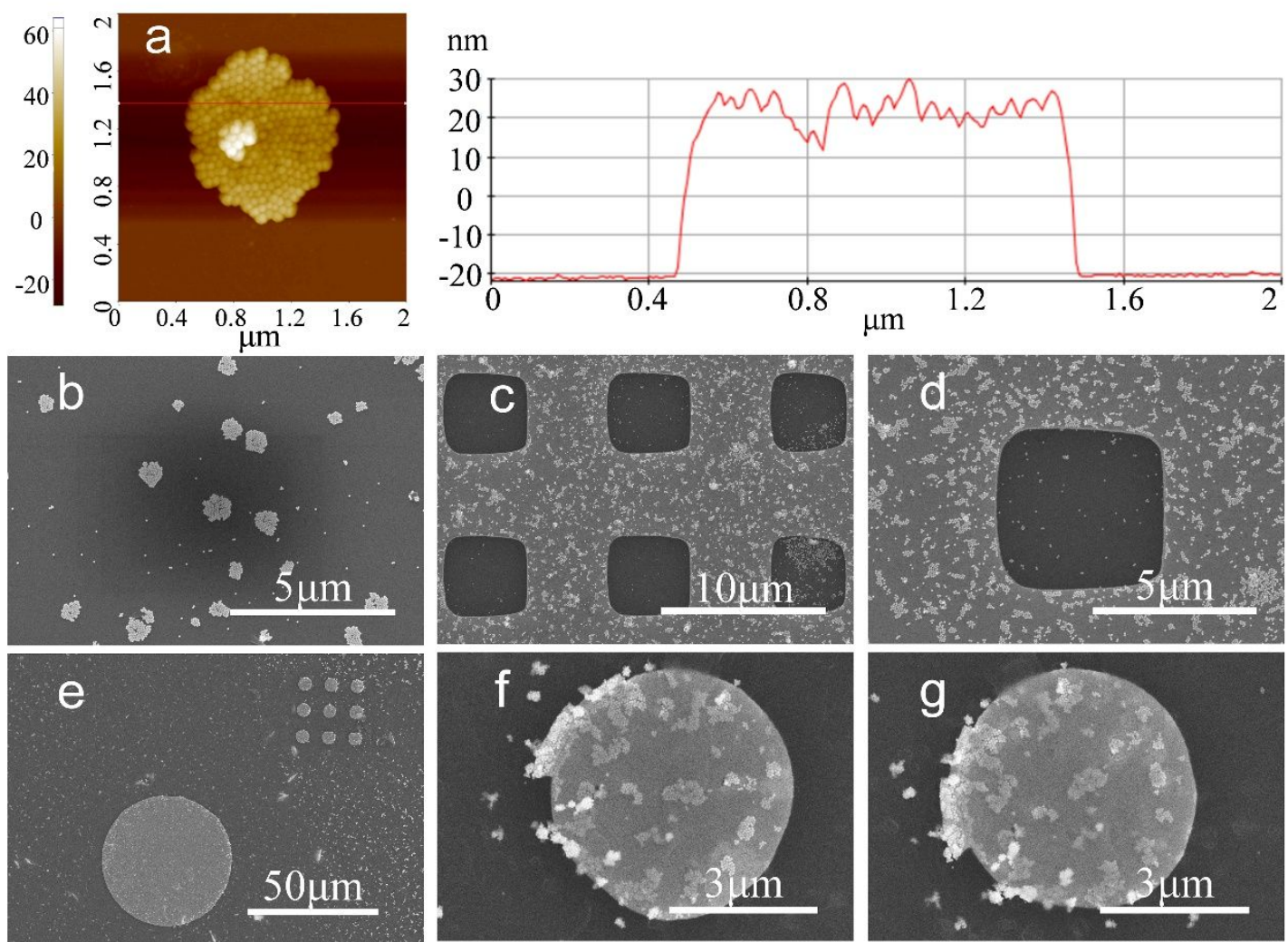

Figure S3. The AFM (a)and SEM images(b-g) of the SERS platform. b, Au NPs on the ITO glass; $\mathrm{c}, \mathrm{d}$ and e, Au NPs on the pattern substrate; $\mathrm{f}$ and $\mathrm{g}$ shows before(f) and after(g) using the $\mathrm{Ar} / \mathrm{H}_{2}$ plasma cleaning for $20 \mathrm{~min}$. 

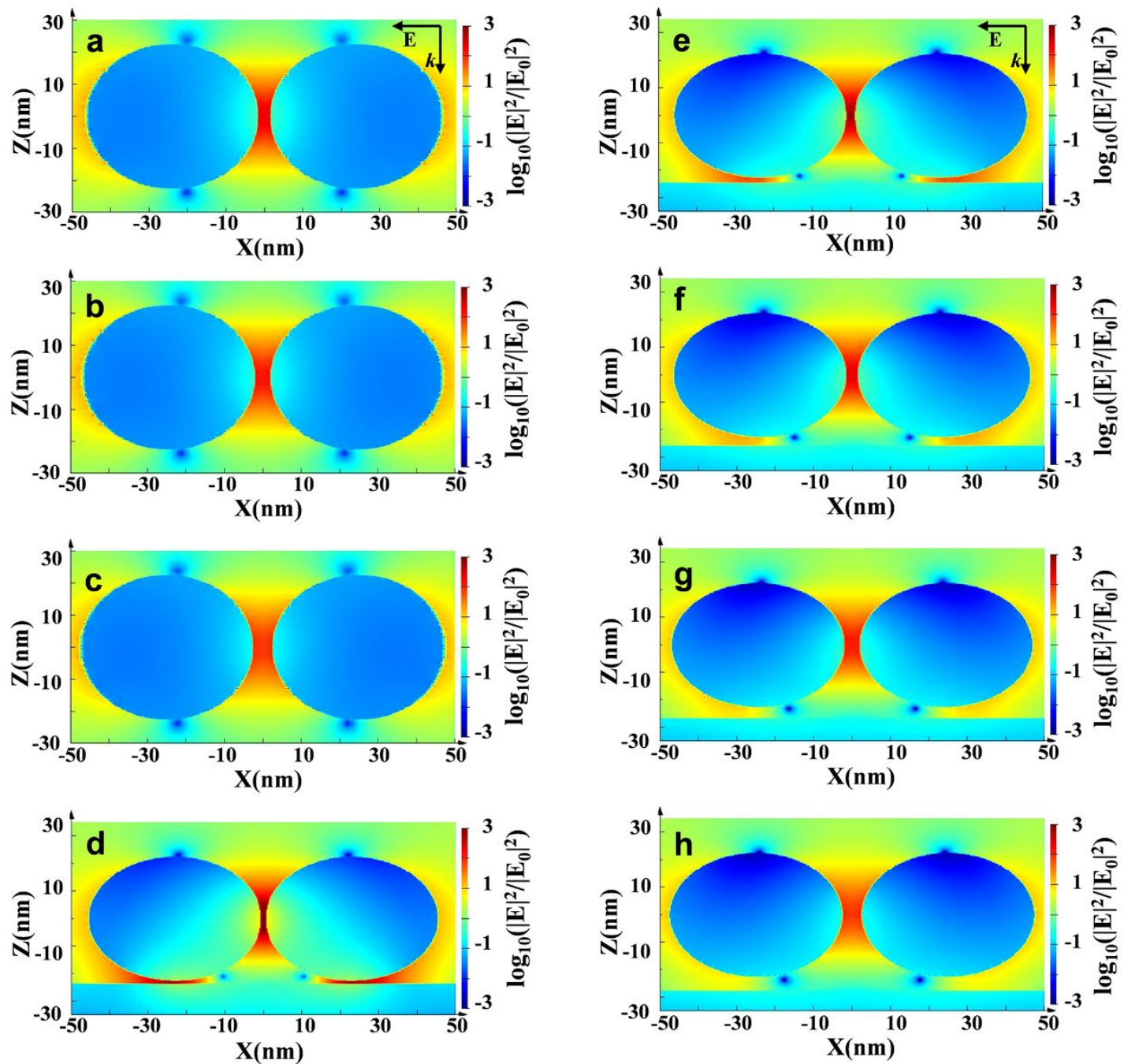

Figure S4. The local electric field intensities of Au nanoparticles computed by FDTD. The modelled two Au nanospheres $(\mathrm{d}=45 \mathrm{~nm})$ : Gap X is set as $3 \mathrm{~nm}(\mathrm{a}), 4 \mathrm{~nm}(\mathrm{~b})$ and $5 \mathrm{~nm}(\mathrm{c})$, respectively. The modelled systems consisting of two Au nanospheres $(\mathrm{d}=$ $45 \mathrm{~nm}$ ) and Au film positioned at different configurations (Gap X = Gap Z); the Gap $\mathrm{X}$ and Gap Z are set as $1 \mathrm{~nm}(\mathrm{~d}), 2 \mathrm{~nm}(\mathrm{e}), 3 \mathrm{~nm}(\mathrm{f}), 4 \mathrm{~nm}(\mathrm{~g})$ and $5 \mathrm{~nm}(\mathrm{~h})$, respectively. 

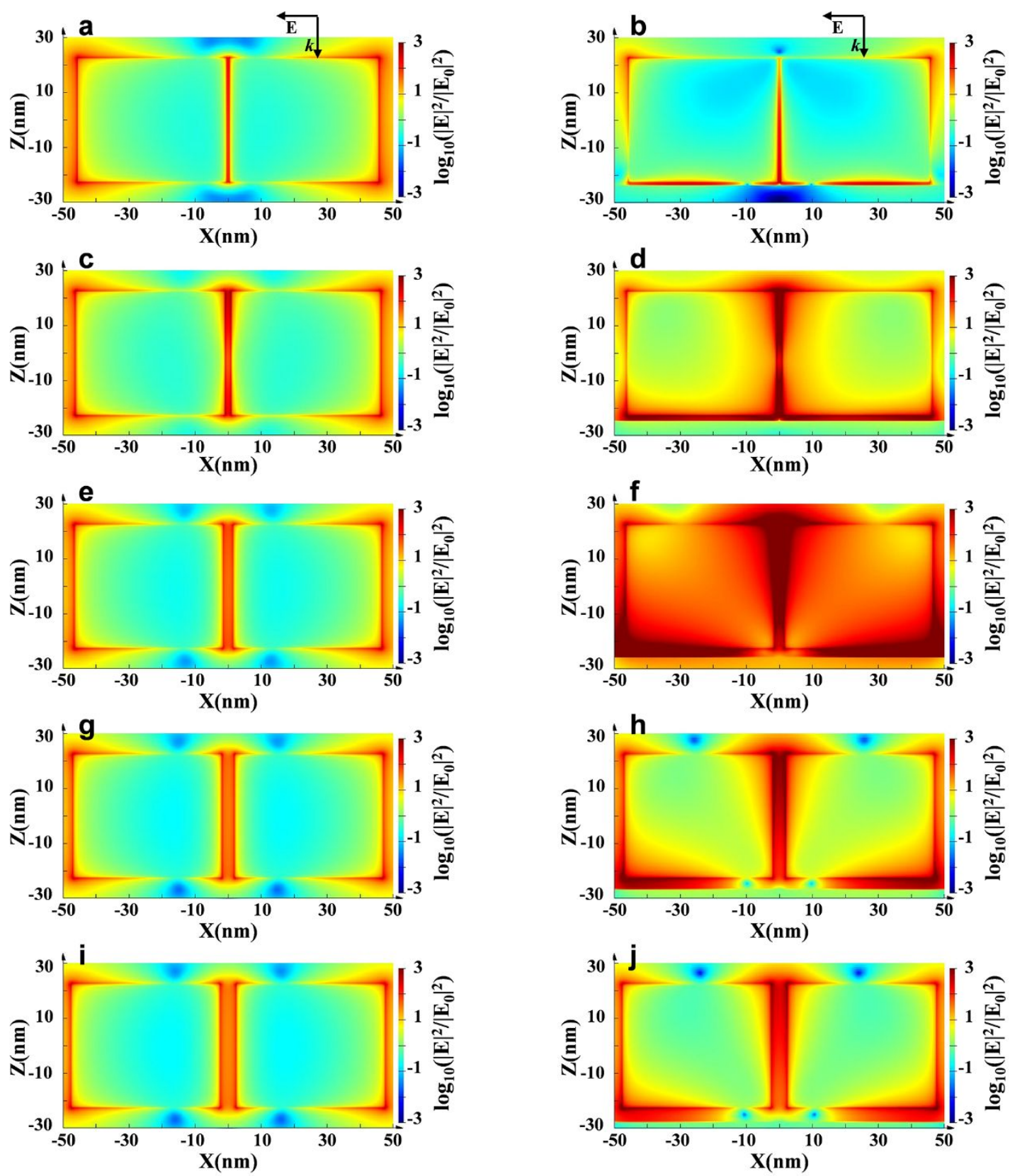

Figure S5. The local near field intensities of Au nanocubes computed by FDTD. The modelled systems consisting of two $\mathrm{Au}$ nanocubes $(\mathrm{a}=45 \mathrm{~nm})$ and $\mathrm{Au}$ film positioned at different configurations (Gap X = Gap Z). The Gap X of the modelled two $\mathrm{Au}$ nanocubes is set as $1 \mathrm{~nm}(\mathrm{a}), 2 \mathrm{~nm}(\mathrm{c}), 3 \mathrm{~nm}(\mathrm{e}), 4 \mathrm{~nm}(\mathrm{~g})$ and $5 \mathrm{~nm}$ (i), respectively. And the Gap X and Gap Z of the Au nanocubes and Au film systems are set as $1 \mathrm{~nm}(\mathrm{~b}), 2 \mathrm{~nm}(\mathrm{~d}), 3 \mathrm{~nm}(\mathrm{f}), 4 \mathrm{~nm}(\mathrm{~h})$ and $5 \mathrm{~nm}(\mathrm{j})$, respectively. 


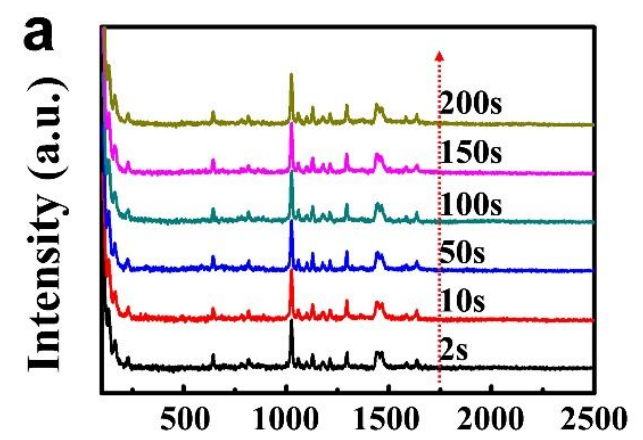

Raman shift $\left(\mathrm{cm}^{-1}\right)$

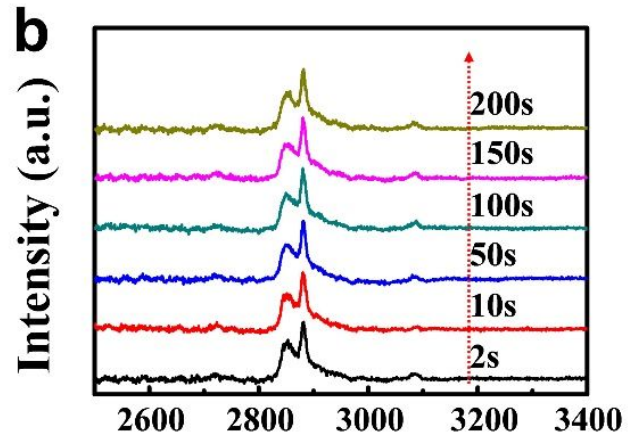

Raman shift $\left(\mathrm{cm}^{-1}\right)$

Figure S6. The Raman curves of CPC power.
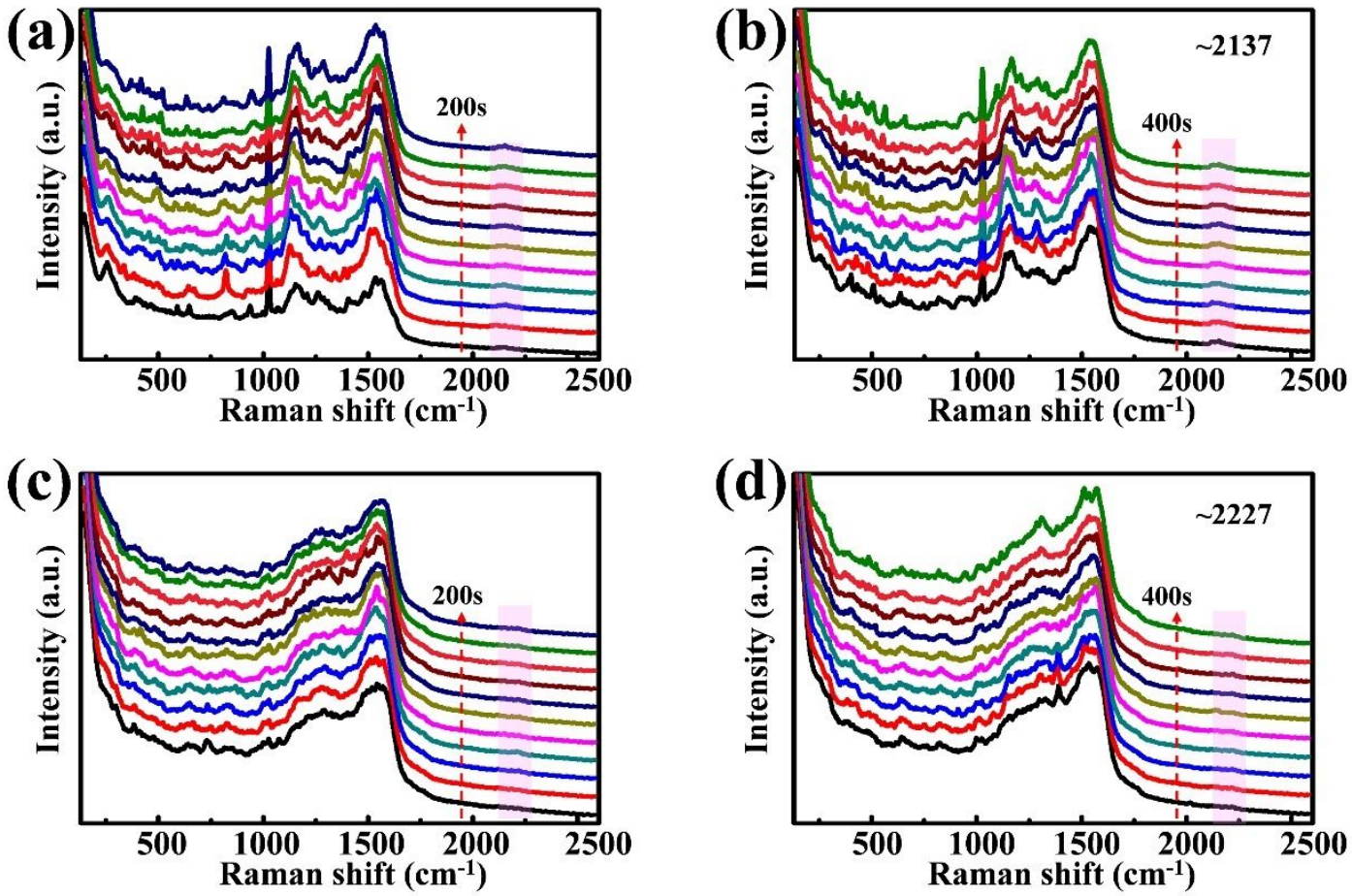

Figure S7. The SERS curves of the systems cleaned by $\mathrm{Ar} / \mathrm{H}_{2}$ plasma, before (a-b) and after 20 min (c-d). Its SEM images are shown in Figure S3. 

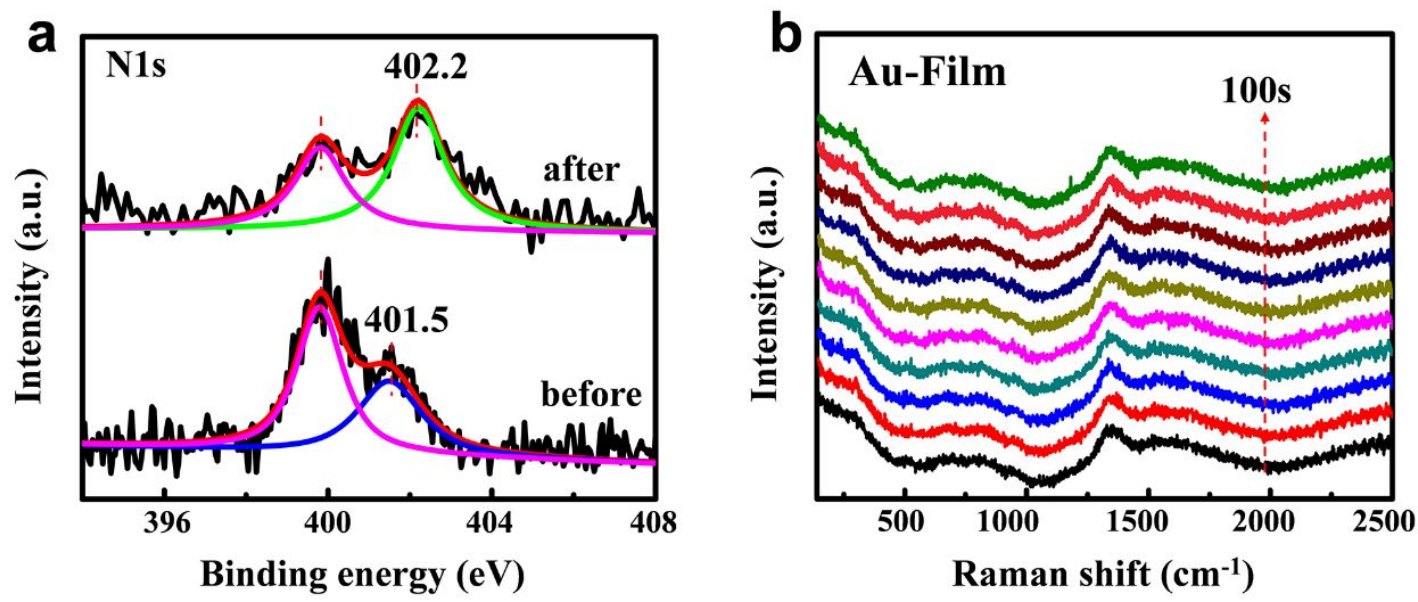

Figure S8. (a) is the measured XPS curves of N1s before and after reaction; (b) is the Raman curves of Au film.
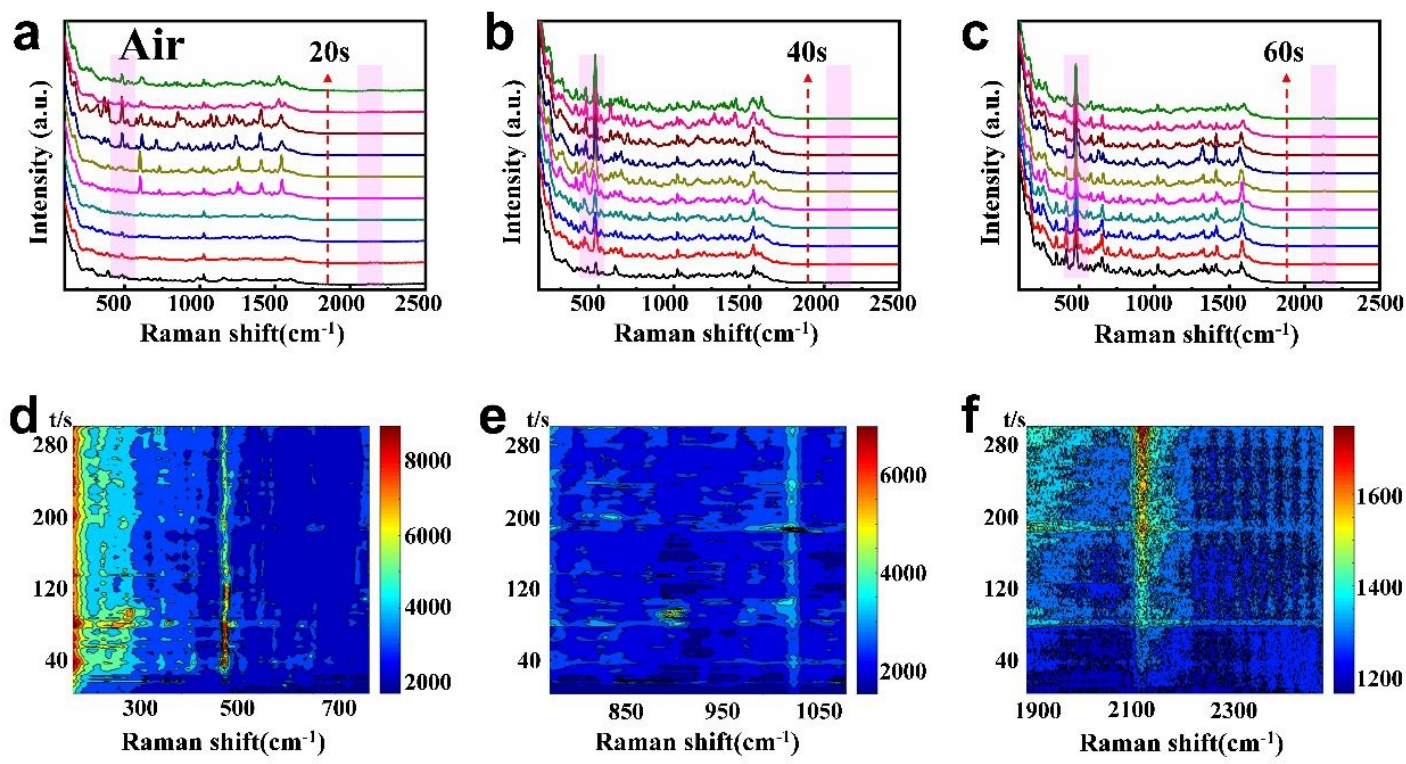

Figure S9. The Raman curves of SRES platform in the air. a-c, the first sixty second; d-e, the changes of SERS peaks intensity from 0 to 300 second. 
Table S1 Mode assignments in $\mathrm{cm}^{-1}$ for $\mathrm{CN}$ species on gold surfaces

\begin{tabular}{|c|c|c|c|c|c|}
\hline $\begin{array}{l}\text { Adsorbed } \\
\text { species }\end{array}$ & Au surface & $v(\mathrm{C} \equiv \mathrm{N})$ & $\delta(\mathrm{Au}-\mathrm{C} \equiv \mathrm{N})$ & $\mathrm{N}(\mathrm{Au}-\mathrm{CN})$ & Ref \\
\hline $\mathrm{CN}^{-}$ & Island film & 2139 & & & 9 \\
\hline $\mathrm{CN}^{-}$ & Island film & 2131 & 291 & 385 & 10 \\
\hline $\mathrm{CN}^{-}$ & Island film & 2181 & 320 & 500 & 10 \\
\hline $\mathrm{CN}^{-}$ & Electrode & $\sim 2100$ & $\sim 300$ & $\sim 370$ & 11 \\
\hline$\left[\mathrm{Au}(\mathrm{CN})_{2}^{-}\right.$ & Colloid & $\sim 2140$ & & & ${ }^{12},^{13}$ \\
\hline$\left[\mathrm{Au}(\mathrm{CN})_{2}\right]^{-}$ & Nanoparticle & $2141^{\mathrm{a}}$ & $298^{a}$ & $400^{\mathrm{a}}$ & 14 \\
\hline$\left[\mathrm{Au}(\mathrm{CN})_{4}\right]^{-}$ & $\begin{array}{l}\text { Colloid/ } \\
\text { Island film }\end{array}$ & 2190 & & & ${ }^{9}, 12$ \\
\hline$\left[\mathrm{Au}(\mathrm{CN})_{4}\right]^{-}$ & Nanoparticle & $2141^{\mathrm{a}}, 2189^{\mathrm{b}}$ & $\sim 289^{\mathrm{c}}$ & $400^{\mathrm{a}}, 539^{\mathrm{b}}$ & 14 \\
\hline$\left[\mathrm{Au}(\mathrm{CN})_{4}\right]^{-}$ & DFT & $2152^{\mathrm{a}}, 2224^{\mathrm{b}}$ & $289^{c}$ & $515^{\mathrm{a}}, 450^{\mathrm{b}}$ & 14 \\
\hline $\begin{array}{l}\text { Undefined } \\
\mathrm{CN}^{-} \text {complex }\end{array}$ & $\begin{array}{l}\text { Particle in a } \\
\text { sol-gel }\end{array}$ & $\sim 2150, \sim 2200$ & & & 15 \\
\hline $\begin{array}{l}\text { Undefined } \\
\mathrm{CN}^{-} \text {complex }\end{array}$ & Particle & 2144,2191 & & & 16 \\
\hline $\mathrm{Au}-\mathrm{CN}$ & Colloid & 2154 & 300 & 370 & 17 \\
\hline $\mathrm{Au}-\mathrm{CN}$ & Particle & $\sim 2227$ & 147,389 & $514,636^{\mathrm{d}}$ & 18 \\
\hline $\mathrm{Au}-\mathrm{CN}$ & $\mathrm{Au}\{111\}$ & $2195^{\mathrm{d}}$ & $141^{\mathrm{d}}, 304$ & 396 & 19 \\
\hline $\mathrm{CN}$ (nitrile) & & $\sim 2044, \sim 2176$ & & & 19 \\
\hline $\mathrm{CN} / \mathrm{Au}$ air ${ }^{\mathrm{e}}$ & & $\begin{array}{c}2140-2165 \\
2235\end{array}$ & 304 & 400 & 20 \\
\hline $\begin{array}{l}\mathrm{Au}-\mathrm{CN} \\
\text { (nitrile) }\end{array}$ & & $\begin{array}{c}\sim 2126-2149, \\
\sim 2220\end{array}$ & $\sim 298$ & $\begin{array}{c}\sim 370-392 \\
458,515\end{array}$ & $\begin{array}{l}\text { In this } \\
\text { work }\end{array}$ \\
\hline
\end{tabular}

$a$, indicates that cyanide groups are bent toward the surface. $b$, indicates that cyanide groups are parallel to the surface. c, indicates two Au-CN bending modes, one for the cyanide groups bent and one for the cyanide groups parallel. $\mathrm{d}$, indicates Au-NC models. e, represents the ATR-FTIR data. 
Table $\mathrm{S} 2^{5,6,8,21,22}$ Vibrational peak frequencies and assignments for pyridine ring and the proposed reaction products

\begin{tabular}{|c|c|c|c|c|c|c|c|c|c|c|c|c|c|c|c|}
\hline \multicolumn{3}{|c|}{$\begin{array}{c}\text { Observed } \\
\text { Frequency }\left(\mathrm{cm}^{-1}\right)\end{array}$} & \multicolumn{3}{|c|}{ Pyridine } & \multicolumn{2}{|c|}{$o$-Pyridyl } & \multicolumn{2}{|c|}{ m-Pyridyl } & \multicolumn{2}{|c|}{$p$-Pyridyl } & \multicolumn{2}{|c|}{ 2,3-Pyridyne } & \multicolumn{2}{|c|}{ 3,4-Pyridyne } \\
\hline $\begin{array}{l}2 s-10 \\
s\end{array}$ & $10 \mathrm{~s}-14 \mathrm{~s}$ & 14-18s & $\begin{array}{c}\text { Frequency } \\
\left(\mathrm{(m}^{-1}\right)\end{array}$ & $\begin{array}{l}\text { Wilson } \\
\text { Number }\end{array}$ & $\begin{array}{l}\text { Vibrational } \\
\text { Mode }\end{array}$ & $\begin{array}{l}\text { Frequency } \\
\left(\mathrm{cm}^{-1}\right)\end{array}$ & $\begin{array}{l}\text { Vibrational } \\
\text { Mode }\end{array}$ & $\begin{array}{l}\text { Frequency } \\
\left(\mathrm{cm}^{-1}\right)\end{array}$ & $\begin{array}{l}\text { Vibrational } \\
\text { Mode }\end{array}$ & $\begin{array}{c}\text { Frequency } \\
\left(\mathrm{cm}^{-1}\right)\end{array}$ & $\begin{array}{l}\text { Vibrational } \\
\text { Mode }\end{array}$ & $\begin{array}{l}\text { Frequency } \\
\left(\mathrm{cm}^{-1}\right)\end{array}$ & $\begin{array}{l}\text { Vibrational } \\
\text { Mode }\end{array}$ & $\begin{array}{l}\text { Frequency } \\
\left(\mathrm{cm}^{-1}\right)\end{array}$ & $\begin{array}{l}\text { Vibrational } \\
\text { Mode }\end{array}$ \\
\hline $\begin{array}{l}332 \\
388\end{array}$ & $\begin{array}{l}363, \\
388,\end{array}$ & 388,407 & 338 & $v_{16 \mathrm{a}}$ & $\delta$ (ring) $)_{\text {oop }}$ & 377 & $\delta(\mathrm{mol})_{\mathrm{oop}}$ & 381 & $\delta(\mathrm{mol})_{\mathrm{oop}}$ & 370 & $\delta(\mathrm{mol})_{\text {oop }}$ & 411 & $\delta(\mathrm{mol})_{\mathrm{oop}}$ & 403 & $\delta(\mathrm{mol})_{\mathrm{oop}}$ \\
\hline $\begin{array}{l}422 \\
572 \\
601\end{array}$ & $\begin{array}{l}453 \\
577, \\
613\end{array}$ & $\begin{array}{c}456 \\
571,607\end{array}$ & $\begin{array}{l}406 \\
603\end{array}$ & $\begin{array}{l}v_{16 b} \\
v_{6 a}\end{array}$ & $\begin{array}{l}\delta(\text { ring })_{\text {oop }} \\
\delta(\text { ring })_{\text {ip }}\end{array}$ & $\begin{array}{l}417 \\
572\end{array}$ & $\begin{array}{l}\delta(\text { ring })_{\text {oop }} \\
\delta(\text { ring })_{\text {ip }}\end{array}$ & $\begin{array}{l}414 \\
572\end{array}$ & $\begin{array}{l}\delta(\text { ring })_{\text {oop }} \\
\delta(\text { ring })_{\text {ip }}\end{array}$ & $\begin{array}{l}436 \\
612\end{array}$ & $\begin{array}{l}\delta(\text { ring })_{\text {oop }} \\
\delta(\text { ring })_{\text {ip }}\end{array}$ & $\begin{array}{l}432 \\
465\end{array}$ & $\begin{array}{c}\delta(\text { ring })_{\text {ip }} \\
\delta(\mathrm{C}-\mathrm{C}-\mathrm{C}-\mathrm{C})_{\text {oop }}\end{array}$ & 451 & $\delta(\mathrm{C}-\mathrm{C})_{\mathrm{ip}}$ \\
\hline & 481 & 481 & & & & & & & & & & & & 478 & $\delta(\mathrm{C}-\mathrm{C})_{\mathrm{oop}}$ \\
\hline 638 & & 627 & & & & & & & & & & & & 637 & $\delta(\mathrm{mol})$ \\
\hline $\begin{array}{l}653 \\
699,\end{array}$ & $\begin{array}{l}674, \\
709\end{array}$ & 674,709 & $\begin{array}{l}654 \\
703\end{array}$ & $\begin{array}{l}v_{6 \mathrm{~b}} \\
v_{11}\end{array}$ & $\begin{array}{c}\delta(\text { ring })_{\text {ip }} \\
\delta(\mathrm{C}-\mathrm{H})_{\text {oop }}\end{array}$ & $\begin{array}{l}655 \\
700\end{array}$ & $\begin{array}{c}\delta(\text { ring })_{\text {ip }} \\
\delta(\mathrm{C}-\mathrm{H})_{\text {oop }}\end{array}$ & $\begin{array}{l}654 \\
674\end{array}$ & $\begin{array}{c}\delta(\text { ring })_{\text {ip }} \\
\delta(\mathrm{C}-\mathrm{H})_{\text {oop }}\end{array}$ & $\begin{array}{l}636 \\
697\end{array}$ & $\begin{array}{c}\delta(\text { ring })_{\text {ip }} \\
\delta(\mathrm{C}-\mathrm{H})_{\text {oop }}\end{array}$ & $\begin{array}{l}651 \\
712\end{array}$ & $\begin{array}{c}\delta(\mathrm{mol}) \\
\delta(\mathrm{mol})_{\mathrm{ip}}\end{array}$ & $\begin{array}{l}658 \\
818\end{array}$ & $\begin{array}{c}\delta(\text { ring }) \\
\delta(\mathrm{C}-\mathrm{H})_{\text {oop }}\end{array}$ \\
\hline $\begin{array}{l}728, \\
810\end{array}$ & $\begin{array}{c}732,760 \\
781\end{array}$ & & 747 & $v_{4}$ & $\delta$ (ring $)_{\text {oop }}$ & 739 & $\delta$ (ring) $)_{\text {oop }}$ & 770 & $\delta$ (ring) $)_{\text {oop }}$ & 754 & $\delta$ (ring) $)_{\text {oop }}$ & 814 & $\delta(\mathrm{C}-\mathrm{H})_{\mathrm{oop}}$ & 848 & $\delta(\mathrm{N}-\mathrm{C}-\mathrm{C})$ \\
\hline 852 & $\begin{array}{l}854,877 \\
898,923\end{array}$ & & 884 & $v_{10 a}$ & $\begin{array}{c}\delta(\mathrm{C}-\mathrm{C})_{\text {oop }} \\
\delta(\mathrm{mol})\end{array}$ & $\begin{array}{l}871 \\
939\end{array}$ & $\begin{array}{c}\delta(\mathrm{C}-\mathrm{H}) \\
\delta(\mathrm{C}-\mathrm{H})_{\text {oop }}\end{array}$ & $\begin{array}{l}902 \\
925\end{array}$ & $\begin{array}{c}\delta(\mathrm{C}-\mathrm{H}) \\
\delta(\mathrm{C}-\mathrm{H})_{\text {oop }}\end{array}$ & $\begin{array}{l}810 \\
940\end{array}$ & $\begin{array}{c}\delta(\mathrm{C}-\mathrm{H}) \\
\delta(\mathrm{C}-\mathrm{H})_{\text {oop }}\end{array}$ & 884 & $\begin{array}{c}\delta(\mathrm{C}-\mathrm{C})_{\text {oop }} \\
\delta(\mathrm{mol})\end{array}$ & $\begin{array}{l}915 \\
944\end{array}$ & $\begin{array}{c}\delta(\mathrm{C}-\mathrm{H}) \\
\delta(\mathrm{C}-\mathrm{H})_{\text {oop }}\end{array}$ \\
\hline 951 & 976 & 930,972 & 941 & $v_{17 \mathrm{~b}}$ & $\delta(\mathrm{C}-\mathrm{H})_{\mathrm{oop}}$ & 950 & $v(\text { ring })_{\text {ip }}$ & 972 & $v(\text { ring })_{\text {ip }}$ & 961 & $v(\text { ring })_{\text {ip }}$ & 926 & $\delta(\mathrm{H}-\mathrm{C}-\mathrm{C}-\mathrm{C}-\mathrm{H})$ & 1009 & $\begin{array}{c}\delta(\mathrm{H}-\mathrm{C}-\mathrm{C}-\mathrm{C}- \\
\mathrm{H})\end{array}$ \\
\hline $\begin{array}{l}986, \\
999\end{array}$ & & & 991 & $v_{1}$ & $v(\mathrm{C}-\mathrm{H})_{\mathrm{ip}}$ & 998 & $v(\text { ring })_{\text {ip }}$ & 973 & $v(\text { ring })_{\text {ip }}$ & 963 & $v(\text { ring })_{\text {ip }}$ & 975 & $\delta(\mathrm{H}-\mathrm{C}-\mathrm{C}-\mathrm{H})$ & & $\delta(\mathrm{H}-\mathrm{C}-\mathrm{C}-\mathrm{H})$ \\
\hline 1024 & 1024 & 1024 & 1030 & $v_{12}$ & $\delta(\text { ring })_{\text {ip }}$ & 1016 & $\delta(\mathrm{C}-\mathrm{H})_{\mathrm{ip}}$ & $\begin{array}{l}1025 \\
1036\end{array}$ & $\begin{array}{l}\delta(\mathrm{C}-\mathrm{H})_{\mathrm{ip}} \\
\delta(\mathrm{C}-\mathrm{H})_{\mathrm{ip}}\end{array}$ & $\begin{array}{l}1020 \\
1045\end{array}$ & $\begin{array}{l}\delta(\mathrm{C}-\mathrm{H})_{\mathrm{ip}} \\
\delta(\mathrm{C}-\mathrm{H})_{\mathrm{ip}}\end{array}$ & 1025 & $\delta(\mathrm{H}-\mathrm{C}-\mathrm{C}-\mathrm{C}-\mathrm{H})$ & & \\
\hline 1051 & 1049 & $\begin{array}{l}1051, \\
1169\end{array}$ & 1052 & $v_{18 b}$ & $\delta(\mathrm{C}-\mathrm{H})_{\mathrm{ip}}$ & 1047 & $\delta(\mathrm{C}-\mathrm{H})_{\mathrm{ip}}$ & 1087 & & 1056 & & 1079 & $\delta(\mathrm{C}-\mathrm{H})$ & 1169 & $\delta(\mathrm{C}-\mathrm{H})_{\mathrm{ip}}$ \\
\hline $\begin{array}{l}1072, \\
1194\end{array}$ & $\begin{array}{l}1068 \\
1194\end{array}$ & 1238 & 1069 & $v_{18 a}$ & & 1093 & & 1177 & $\delta(\mathrm{C}-\mathrm{H})_{\mathrm{ip}}$ & 1209 & $\delta(\mathrm{C}-\mathrm{H})_{\mathrm{ip}}$ & 1082 & $\delta(\mathrm{mol})_{\mathrm{ip}}$ & 1247 & $\delta(\mathrm{C}-\mathrm{H})_{\mathrm{ip}}$ \\
\hline 1154 & $\begin{array}{l}1125, \\
1178\end{array}$ & $\begin{array}{l}1129, \\
1150\end{array}$ & 1146 & $v_{15}$ & $\delta(\mathrm{C}-\mathrm{H})$ & 1150 & $\delta(\mathrm{C}-\mathrm{H})_{\mathrm{ip}}$ & 1222 & $\delta(\mathrm{C}-\mathrm{H})_{\mathrm{ip}}$ & 1217 & $\delta(\mathrm{C}-\mathrm{H})_{\mathrm{ip}}$ & 1134 & $\delta(\mathrm{H}-\mathrm{C}-\mathrm{C}-\mathrm{H})$ & 1292 & $\delta(\mathrm{C}-\mathrm{H})_{\mathrm{ip}}$ \\
\hline $\begin{array}{l}\text { 1251, } \\
1270\end{array}$ & $\begin{array}{l}1238, \\
1290\end{array}$ & & 1217 & $v_{9 a}$ & $v(\text { ring })_{\text {ip }}$ & 1228 & $\delta(\mathrm{C}-\mathrm{H})_{\mathrm{ip}}$ & 1294 & $\delta(C-H)_{i p}$ & 1291 & $\delta(\mathrm{C}-\mathrm{H})_{\mathrm{ip}}$ & 1156 & $\delta(\mathrm{H}-\mathrm{C}-\mathrm{C}-\mathrm{H})$ & 1387 & $\delta(\mathrm{C}-\mathrm{H})$ \\
\hline \multirow[t]{3}{*}{1408} & $\begin{array}{l}1370, \\
1400\end{array}$ & & 1227 & $v_{14}$ & $\delta(\mathrm{C}-\mathrm{H})_{\mathrm{ip}}$ & 1305 & $\delta(\mathrm{C}-\mathrm{H})_{\mathrm{ip}}$ & 1407 & $\delta(\mathrm{C}-\mathrm{H})$ & 1377 & $\delta(\mathrm{C}-\mathrm{H})$ & 1317 & $\delta(\mathrm{H}-\mathrm{C}-\mathrm{C}-\mathrm{C}-\mathrm{H})_{\mathrm{i}}$ & 1397 & $\delta(\mathrm{mol})$ \\
\hline & $\begin{array}{l}1326, \\
1370\end{array}$ & & 1355 & $v_{3}$ & $\delta(\mathrm{C}-\mathrm{H})_{\mathrm{ip}}$ & 1389 & $\delta(\mathrm{C}-\mathrm{H})$ & & & & & & & & \\
\hline & 1450 & 1400 & 1437 & $v_{19 b}$ & $\delta(\mathrm{C}-\mathrm{H})_{\mathrm{ip}}$ & 1464 & $\delta(\mathrm{C}-\mathrm{H})_{\mathrm{ip}}$ & 1434 & $\delta(\mathrm{C}-\mathrm{H})_{\mathrm{ip}}$ & 1436 & $\delta(\mathrm{C}-\mathrm{H})_{\mathrm{ip}}$ & 1410 & $\delta(\mathrm{C}-\mathrm{H})$ & 1457 & $\delta(\mathrm{C}-\mathrm{H})$ \\
\hline 1496 & 1496 & 1446 & 1483 & $v_{19 a}$ & $\delta(\mathrm{C}-\mathrm{H})_{\mathrm{ip}}$ & 1526 & $\delta(\mathrm{C}-\mathrm{H})_{\mathrm{ip}}$ & 1495 & $\delta(\mathrm{C}-\mathrm{H})_{\mathrm{ip}}$ & 1491 & $\delta(\mathrm{C}-\mathrm{H})_{\mathrm{ip}}$ & 1445 & $\delta(\mathrm{H}-\mathrm{C}-\mathrm{C}-\mathrm{H})_{\mathrm{ip}}$ & & \\
\hline \multirow[t]{2}{*}{1545} & 1543 & & $\begin{array}{l}1574 \\
1581\end{array}$ & $\begin{array}{l}v_{8 b} \\
v_{8 a}\end{array}$ & $\begin{array}{l}v(\text { ring })_{\text {ip }} \\
v(\text { ring })_{\text {in }}\end{array}$ & 1612 & $v(\text { ring })_{\text {ip }}$ & 1564 & $v(\text { ring })_{\text {ip }}$ & 1574 & $v(\text { ring })_{\text {ip }}$ & 1474 & & & \\
\hline & & & & & & & & & & & & 1863 & $v(\mathrm{C} \equiv \mathrm{C})_{\mathrm{ip}}$ & 1917 & $v(\mathrm{C} \equiv \mathrm{C})_{\mathrm{ip}}$ \\
\hline
\end{tabular}


Table $\mathrm{S} 3^{8}$ Vibrational peak frequencies and assignments for the proposed reaction

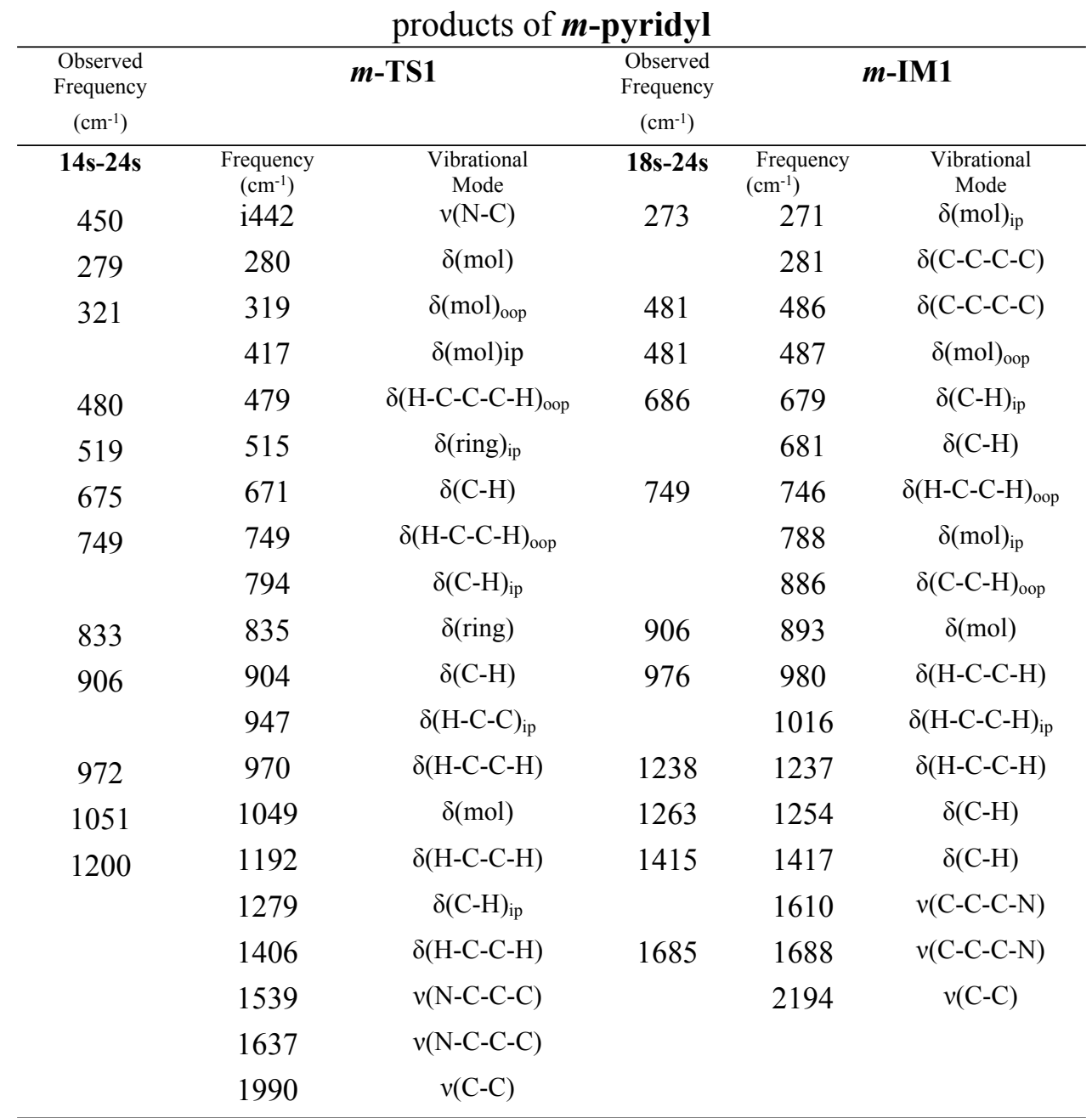


Table $\mathrm{S} 4^{8}$ Vibrational peak frequencies and assignments for the proposed reaction products of $\boldsymbol{m}$-pyridyl.

\begin{tabular}{|c|c|c|c|c|c|c|c|c|c|c|c|c|c|c|}
\hline \multirow{2}{*}{$\begin{array}{c}\text { Observed } \\
\text { Frequency } \\
\left(\mathrm{cm}^{-1}\right)\end{array}$} & \multicolumn{2}{|c|}{$m$-TS4 } & \multirow{2}{*}{$\begin{array}{c}\begin{array}{c}\text { Observed } \\
\text { Frequency } \\
\left(\mathrm{cm}^{-1}\right)\end{array} \\
\mathbf{2 4 - 3 2 s}\end{array}$} & \multicolumn{2}{|c|}{$m$-IM3 } & \multirow{2}{*}{$\begin{array}{c}\begin{array}{c}\text { Observed } \\
\text { Frequency } \\
\left(\mathrm{cm}^{-1}\right)\end{array} \\
\mathbf{2 4 - 3 2 s}\end{array}$} & \multicolumn{2}{|c|}{$m$-TS2 } & \multirow{2}{*}{$\begin{array}{c}\begin{array}{c}\text { Observed } \\
\text { Frequency } \\
\left(\mathrm{cm}^{-1}\right)\end{array} \\
\mathbf{2 4 - 3 2 s}\end{array}$} & \multicolumn{2}{|c|}{$m$-IM2 } & \multirow{2}{*}{$\begin{array}{c}\text { Observed } \\
\begin{array}{c}\text { Frequency } \\
\left(\mathrm{cm}^{-1}\right)\end{array} \\
\mathbf{2 4 - 3 2 s}\end{array}$} & \multicolumn{2}{|c|}{ m-TS3 } \\
\hline & $\begin{array}{c}\text { Frequen } \\
\text { cy } \\
\left(\mathrm{cm}^{-1}\right)\end{array}$ & $\begin{array}{l}\text { Vibrational } \\
\text { Mode }\end{array}$ & & $\begin{array}{l}\text { Frequency } \\
\left(\mathrm{cm}^{-1}\right)\end{array}$ & $\begin{array}{l}\text { Vibrational } \\
\text { Mode }\end{array}$ & & $\begin{array}{l}\text { Frequency } \\
\left(\mathrm{cm}^{-1}\right)\end{array}$ & $\begin{array}{l}\text { Vibrational } \\
\text { Mode }\end{array}$ & & $\begin{array}{l}\text { Frequency } \\
\left(\mathrm{cm}^{-1}\right)\end{array}$ & $\begin{array}{l}\text { Vibrational } \\
\text { Mode }\end{array}$ & & $\begin{array}{l}\text { Frequency } \\
\left(\mathrm{cm}^{-1}\right)\end{array}$ & $\begin{array}{l}\text { Vibrational } \\
\text { Mode }\end{array}$ \\
\hline 806 & i810 & $v(\mathrm{C}-\mathrm{H})$ & & & & 351 & i346 & $v(\mathrm{C}-\mathrm{C})$ & & 227 & $\delta(\mathrm{mol})$ & 900 & i897 & $v(\mathrm{C}-\mathrm{H})$ \\
\hline 240 & 240 & $\delta(\mathrm{mol})$ & 235 & 231 & $\delta(\mathrm{mol})$ & & 206 & $\delta(\mathrm{mol})_{\mathrm{ip}}$ & & 330 & $\delta(\mathrm{mol})_{\text {oop }}$ & 261 & 258 & $\delta(\mathrm{mol})_{\text {oop }}$ \\
\hline 286 & 283 & $\delta(\mathrm{mol})$ & 258 & 264 & $\delta(\mathrm{mol})_{\mathrm{ip}}$ & & 308 & $\delta(\mathrm{mol})$ & & 532 & $\delta(\mathrm{mol})_{\mathrm{ip}}$ & & 265 & $\delta(\mathrm{mol})$ \\
\hline \multirow[t]{2}{*}{384} & 392 & $\delta(\mathrm{mol})_{\text {oop }}$ & 378 & 372 & $\delta(\mathrm{mol})_{\text {oop }}$ & 351 & 358 & $\delta(\mathrm{C}-\mathrm{H})_{\text {oop }}$ & 655 & 650 & $\delta(\mathrm{C}-\mathrm{C}-\mathrm{H})$ & & 464 & $\delta(\mathrm{C}-\mathrm{H}) \mathrm{i}_{\mathrm{p}}$ \\
\hline & 499 & $\delta(\mathrm{C}-\mathrm{C}-\mathrm{C}-\mathrm{H})$ & 468 & 466 & $\delta(\mathrm{C}-\mathrm{C}-\mathrm{C}-\mathrm{C})$ & 579 & 579 & $\delta(\mathrm{C}-\mathrm{H}) \mathrm{i}_{\mathrm{p}}$ & 669 & 671 & $\delta(\mathrm{C}-\mathrm{H})$ & & 536 & $\delta(\mathrm{mol})$ \\
\hline 554 & 561 & $\delta(\mathrm{C}-\mathrm{H})_{\mathrm{ip}}$ & 611 & 614 & $\delta(\mathrm{C}-\mathrm{C}-\mathrm{C}-\mathrm{C})$ & 620 & 623 & $\delta(\mathrm{C}-\mathrm{C}-\mathrm{H})$ & 690 & 692 & $\delta(\mathrm{C}-\mathrm{C}-\mathrm{H})$ & 551 & 547 & $\delta(\mathrm{H}-\mathrm{C}-\mathrm{C}-\mathrm{C})$ \\
\hline 620 & 620 & $\delta(\mathrm{mol})$ & 691 & 692 & $\delta(\mathrm{mol})$ & 651 & 646 & $\delta(\mathrm{C}-\mathrm{C}-\mathrm{H})_{\mathrm{ip}}$ & 804 & 796 & $\delta(\mathrm{C}-\mathrm{H}) \mathrm{i}_{\mathrm{p}}$ & 661 & 663 & $\delta(\mathrm{H}-\mathrm{C}-\mathrm{C}-\mathrm{H})_{\mathrm{i}}$ \\
\hline \multirow[t]{3}{*}{691} & 692 & $\delta(\mathrm{C}-\mathrm{H})_{\mathrm{ip}}$ & 753 & 742 & $\begin{array}{c}\delta(\mathrm{C}-\mathrm{C}-\mathrm{C}-\mathrm{C}-\mathrm{C}- \\
\mathrm{N})\end{array}$ & 686 & 682 & $\delta(\mathrm{C}-\mathrm{C}-\mathrm{C}-\mathrm{H})_{\text {oop }}$ & 830 & 834 & $\delta(\mathrm{H}-\mathrm{C}-\mathrm{C}-\mathrm{H})$ & & 667 & $\begin{array}{c}\mathrm{p} \\
\delta(\mathrm{C}-\mathrm{H})\end{array}$ \\
\hline & 695 & $\delta(\mathrm{C}-\mathrm{H})$ & 774 & 774 & $\delta(\mathrm{H}-\mathrm{C}-\mathrm{C}-\mathrm{H})$ & 727 & 735 & $\delta(\mathrm{H}-\mathrm{C}-\mathrm{N})_{\mathrm{oop}}$ & 1002 & 1009 & $\delta(\mathrm{H}-\mathrm{C}-\mathrm{C}-\mathrm{H})$ & & 669 & $\delta(\mathrm{C}-\mathrm{H}) \mathrm{i}_{\mathrm{p}}$ \\
\hline & 763 & $\delta(\mathrm{C}-\mathrm{C}-\mathrm{C}-\mathrm{C})$ & 883 & 889 & $\begin{array}{c}\delta(\mathrm{C}-\mathrm{C}-\mathrm{C}-\mathrm{C}-\mathrm{C}- \\
\mathrm{N})_{\mathrm{ip}}\end{array}$ & 751 & 759 & $\delta(\mathrm{H}-\mathrm{C}-\mathrm{N})_{\mathrm{ip}}$ & 1260 & 1260 & $\delta(\mathrm{C}-\mathrm{H})$ & & 677 & $\delta(\mathrm{C}-\mathrm{H})$ \\
\hline 774 & 775 & $\delta(\mathrm{H}-\mathrm{C}-\mathrm{C}-\mathrm{H})$ & 986 & 986 & $\delta(\mathrm{C}-\mathrm{C}-\mathrm{C}-\mathrm{H})$ & 838 & 835 & $\delta(\mathrm{C}-\mathrm{H})$ & 1638 & 1635 & $v(\mathrm{C}-\mathrm{C})$ & & 915 & $\delta(\mathrm{C}-\mathrm{C}-\mathrm{C}-\mathrm{C})$ \\
\hline 883 & 890 & $\delta(\mathrm{mol}) \mathrm{pp}$ & 1047 & 1035 & $\delta(\mathrm{C}-\mathrm{C}-\mathrm{C}-\mathrm{C})$ & 871 & 874 & $\delta(\mathrm{C}-\mathrm{C}-\mathrm{C}-\mathrm{H})_{\mathrm{ip}}$ & & 2204 & $v(\mathrm{C}-\mathrm{C})$ & & 2038 & $v(\mathrm{C}-\mathrm{C})$ \\
\hline 986 & 984 & $\delta(\mathrm{H}-\mathrm{C}-\mathrm{C}-\mathrm{H})$ & 1242 & 1241 & $\delta(\mathrm{H}-\mathrm{C}-\mathrm{C}-\mathrm{H})$ & 1052 & 1057 & $\delta(\mathrm{H}-\mathrm{C}-\mathrm{C}-\mathrm{H})$ & & & & & 2217 & $v(\mathrm{C}-\mathrm{C})$ \\
\hline 1024 & 1029 & $\delta(\mathrm{mol})$ & 1410 & 1414 & $\delta(\mathrm{H}-\mathrm{C}-\mathrm{C}-\mathrm{H})$ & 1272 & 1270 & $\delta(\mathrm{C}-\mathrm{H})$ & & & & & & \\
\hline 1242 & 1244 & $\delta(\mathrm{H}-\mathrm{C}-\mathrm{C}-\mathrm{H})$ & 1638 & 1639 & $v(\mathrm{C}-\mathrm{C})$ & & 1653 & $v(\mathrm{C}-\mathrm{C})$ & & & & & & \\
\hline \multirow[t]{4}{*}{1410} & 1411 & $\delta(\mathrm{H}-\mathrm{C}-\mathrm{C}-\mathrm{H})$ & & 2202 & $v(\mathrm{C}-\mathrm{C})$ & & 2082 & $v(\mathrm{C}-\mathrm{N})$ & & & & & & \\
\hline & 1626 & $v(\mathrm{C}-\mathrm{C})$ & & 2327 & $v(\mathrm{C}-\mathrm{N})$ & & 2227 & & & & & & & \\
\hline & 2190 & $v(\mathrm{C}-\mathrm{C})$ & & & & & & & & & & & & \\
\hline & 2209 & $v(\mathrm{C}-\mathrm{N})$ & & & & & & & & & & & & \\
\hline
\end{tabular}


Table $\mathrm{S} 5^{5}$ Vibrational peak frequencies and assignments for the proposed reaction products of $\boldsymbol{p}$-pyridyl.

\begin{tabular}{|c|c|c|c|c|c|c|c|c|c|c|c|}
\hline $\begin{array}{c}\text { Observed } \\
\text { Frequency } \\
\left(\mathrm{cm}^{-1}\right)\end{array}$ & & -TS1 & $\begin{array}{c}\text { Observed } \\
\text { Frequency } \\
\left(\mathrm{cm}^{-1}\right)\end{array}$ & & $p$-IM1 & $\begin{array}{c}\text { Observed } \\
\text { Frequency } \\
\left(\mathrm{cm}^{-1}\right)\end{array}$ & & $p$-IM2 & $\begin{array}{c}\text { Observed } \\
\text { Frequency } \\
\left(\mathrm{cm}^{-1}\right)\end{array}$ & & $p$-TS2 \\
\hline $\begin{array}{c}14-18 s \\
407\end{array}$ & $\begin{array}{c}\begin{array}{c}\text { Frequency } \\
\left(\mathrm{cm}^{-1}\right)\end{array} \\
\text { i405 } \\
210\end{array}$ & $\begin{array}{c}\text { Vibrational } \\
\text { Mode } \\
v(\mathrm{C}-\mathrm{C}) \\
\delta(\mathrm{mol})\end{array}$ & $18-24 s$ & $\begin{array}{c}\begin{array}{c}\text { Frequency } \\
\left(\mathrm{cm}^{-1}\right) \\
1937 \\
207\end{array}\end{array}$ & $\begin{array}{c}\text { Vibrational } \\
\text { Mode } \\
\delta(\mathrm{H}-\mathrm{C}-\mathrm{N})_{\text {oop }} \\
\delta(\mathrm{mol})\end{array}$ & $24-32 s$ & $\begin{array}{c}\begin{array}{c}\text { Frequency } \\
\left(\mathrm{cm}^{-1}\right) \\
227 \\
330\end{array}\end{array}$ & $\begin{array}{c}\text { Vibrational } \\
\text { Mode } \\
\delta(\mathrm{mol}) \\
\delta(\mathrm{mol})_{\text {oop }}\end{array}$ & 260 & $\begin{array}{c}\begin{array}{c}\text { Frequency } \\
\left(\mathrm{cm}^{-1}\right)\end{array} \\
1897 \\
258\end{array}$ & $\begin{array}{c}\text { Vibrational } \\
\text { Mode } \\
v(\mathrm{C}-\mathrm{H}) \\
\delta(\mathrm{mol})_{\text {oop }}\end{array}$ \\
\hline 279 & 266 & $\delta(\mathrm{H}-\mathrm{C}-\mathrm{N}-\mathrm{C})$ & 244 & 244 & $\delta(\mathrm{mol})_{\mathrm{ip}}$ & & 532 & $\delta(\mathrm{mol})_{\mathrm{ip}}$ & & 265 & $\delta(\mathrm{mol})$ \\
\hline 388 & 388 & $\delta(\mathrm{mol})_{\mathrm{ip}}$ & 348 & 348 & $\delta(\mathrm{mol})_{\text {oop }}$ & 653 & 650 & $\delta(\mathrm{C}-\mathrm{C}-\mathrm{H})$ & 468 & 464 & $\delta(\mathrm{C}-\mathrm{H})_{\mathrm{ip}}$ \\
\hline 424 & 427 & $\delta(\mathrm{mol})_{\mathrm{oop}}$ & & 443 & $\delta(\mathrm{C}-\mathrm{C}-\mathrm{C}-\mathrm{C})$ & 691 & 671 & $\delta(\mathrm{C}-\mathrm{H})$ & 554 & 536 & $\delta(\mathrm{mol})$ \\
\hline 481 & 474 & $\delta(\mathrm{N}-\mathrm{C}-\mathrm{C}-\mathrm{C})$ & 456 & 453 & $\delta(\mathrm{C}-\mathrm{C}-\mathrm{C}-\mathrm{N})$ & 806 & 692 & $\delta(\mathrm{C}-\mathrm{C}-\mathrm{H})_{\mathrm{ip}}$ & & 547 & $\delta(\mathrm{H}-\mathrm{C}-\mathrm{C}-\mathrm{C})$ \\
\hline 627 & 626 & $\delta(\mathrm{C}-\mathrm{H})$ & 481 & 480 & $\delta(\mathrm{C}-\mathrm{C}-\mathrm{H})_{\mathrm{oop}}$ & 839 & 796 & $\delta(\mathrm{C}-\mathrm{H})_{\mathrm{ip}}$ & 669 & 663 & $\delta(\mathrm{H}-\mathrm{C}-\mathrm{C}-\mathrm{H})_{\text {ip }}$ \\
\hline 674 & 673 & $\begin{array}{c}\delta(\mathrm{H}-\mathrm{C}-\mathrm{N}-\mathrm{C}-\mathrm{H} \\
)_{\mathrm{oop}}\end{array}$ & 611 & 611 & $\delta(\mathrm{H}-\mathrm{C}-\mathrm{C}-\mathrm{H})_{\mathrm{oo}}$ & & 834 & $\delta(\mathrm{H}-\mathrm{C}-\mathrm{C}-\mathrm{H})$ & & 667 & $\delta(\mathrm{C}-\mathrm{H})$ \\
\hline 728 & 730 & $\delta(\mathrm{C}-\mathrm{H})_{\mathrm{ip}}$ & 686 & 687 & $\delta(\mathrm{C}-\mathrm{H})_{\mathrm{ip}}$ & 1257 & 1009 & $\delta(\mathrm{H}-\mathrm{C}-\mathrm{C}-\mathrm{H})$ & & 669 & $\delta(\mathrm{C}-\mathrm{H})_{\mathrm{ip}}$ \\
\hline & 762 & $\delta(\mathrm{N}-\mathrm{C}-\mathrm{C}-\mathrm{C})_{\mathrm{ip}}$ & 728 & 730 & $\delta(\mathrm{H}-\mathrm{C}-\mathrm{C}-\mathrm{H})$ & & 1260 & $\delta(\mathrm{C}-\mathrm{H})$ & & 677 & $\delta(\mathrm{C}-\mathrm{H})$ \\
\hline & 784 & $\delta(\mathrm{C}-\mathrm{H})$ & 749 & 753 & $\delta(\mathrm{H}-\mathrm{C}-\mathrm{N}-\mathrm{C})_{\mathrm{ip}}$ & & 1635 & $v(\mathrm{C}-\mathrm{C})$ & 911 & 915 & $\delta(\mathrm{C}-\mathrm{C}-\mathrm{C}-\mathrm{C})$ \\
\hline & 947 & $\delta(\mathrm{mol})$ & & 767 & $\delta(\mathrm{C}-\mathrm{H})_{\mathrm{ip}}$ & & 2204 & $v(\mathrm{C}-\mathrm{C})$ & & 2038 & $v(\mathrm{C}-\mathrm{C})$ \\
\hline & 960 & $\delta(\mathrm{H}-\mathrm{C}-\mathrm{C}-\mathrm{H})$ & & 917 & $\delta(\mathrm{C}-\mathrm{C}-\mathrm{C}-\mathrm{C})_{\mathrm{ip}}$ & & & & & 2217 & $v(\mathrm{C}-\mathrm{C})$ \\
\hline 1051 & 1063 & $\delta(\mathrm{C}-\mathrm{C}-\mathrm{C})_{\mathrm{ip}}$ & & 1096 & $\delta(\mathrm{C}-\mathrm{H})$ & & & & & & \\
\hline & 1116 & $\delta(\mathrm{C}-\mathrm{H})$ & & 1220 & $\delta(\mathrm{C}-\mathrm{H})$ & & & & & & \\
\hline 1238 & 1236 & $\delta(\mathrm{H}-\mathrm{C}-\mathrm{C}-\mathrm{H})$ & 1410 & 1404 & $\delta(\mathrm{H}-\mathrm{C}-\mathrm{C}-\mathrm{H})$ & & & & & & \\
\hline 1415 & 1414 & $\delta(\mathrm{H}-\mathrm{C}-\mathrm{C}-\mathrm{H})_{\mathrm{ip}}$ & & 1462 & $v(\mathrm{C}-\mathrm{C})$ & & & & & & \\
\hline & 1545 & $v(\mathrm{C}-\mathrm{C})$ & & 2099 & $v(\mathrm{C}-\mathrm{C})$ & & & & & & \\
\hline 1685 & 1702 & $v(\mathrm{~N}-\mathrm{C})$ & & 2133 & $v(\mathrm{C}-\mathrm{N})$ & & & & & & \\
\hline
\end{tabular}




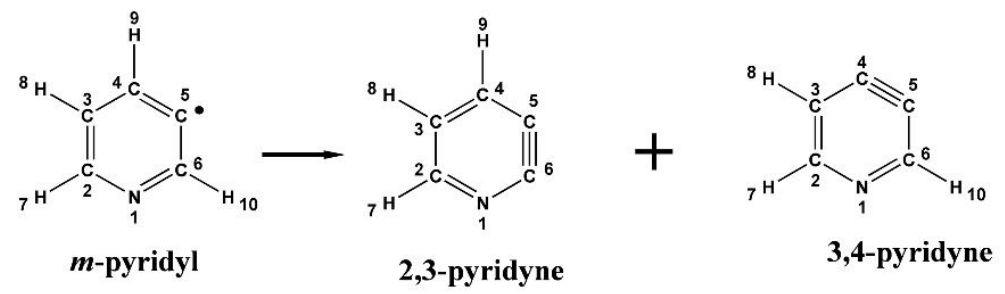<smiles>CC#C/C=C\C(=N)/C=C\C#CC</smiles>

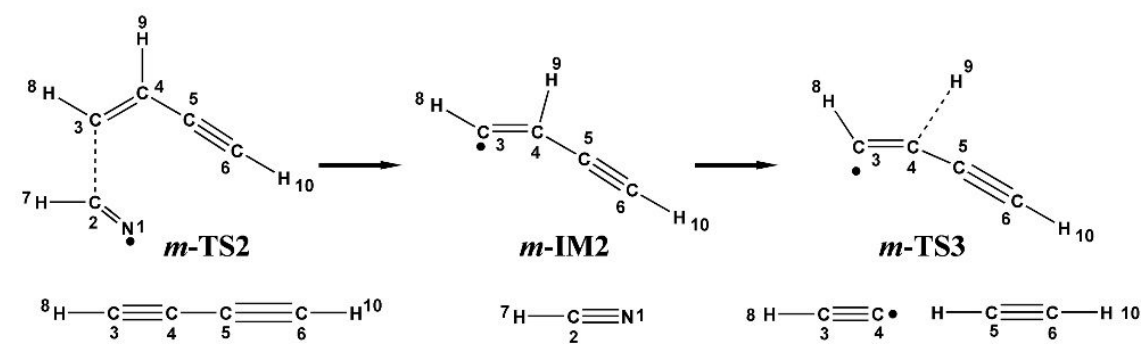

Figure S10. The reaction pathways and products of $\boldsymbol{m}$-pyridyl.
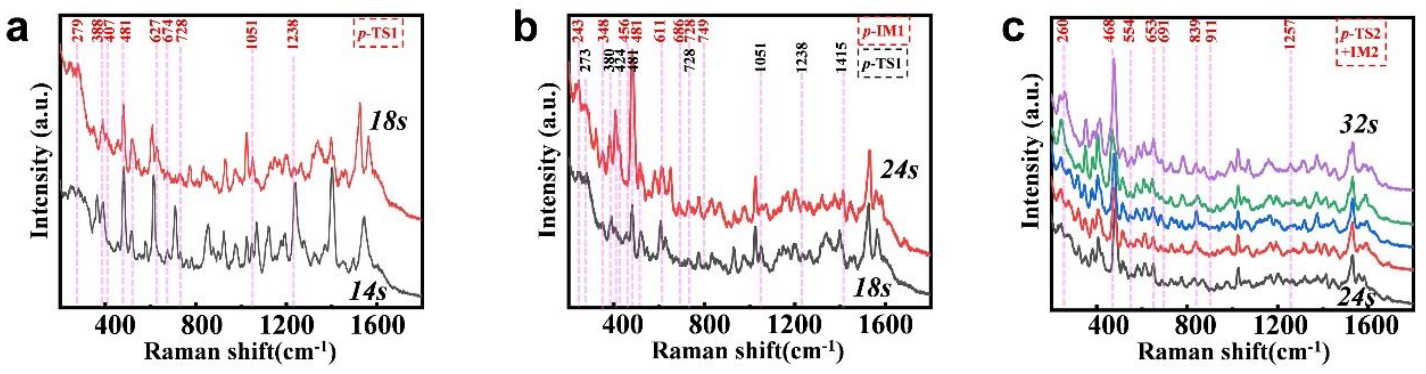

Figure S11. The SERS curves of the reaction products of $\boldsymbol{p}$-pyridyl. 
<smiles>[2H]c1ccccc1</smiles>

p-pyridyl

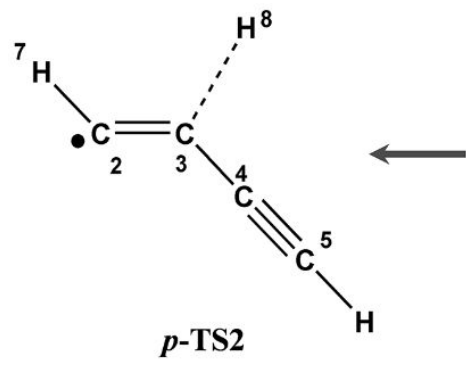

$\underset{7}{\mathrm{H}}-\underset{2}{\mathrm{C}} \equiv \underset{3}{\mathrm{C}}-\underset{4}{\mathrm{C}} \equiv \mathrm{C}-\mathrm{H}$<smiles>[1H]c1c#ccnc1</smiles>

3,4-pyridyne
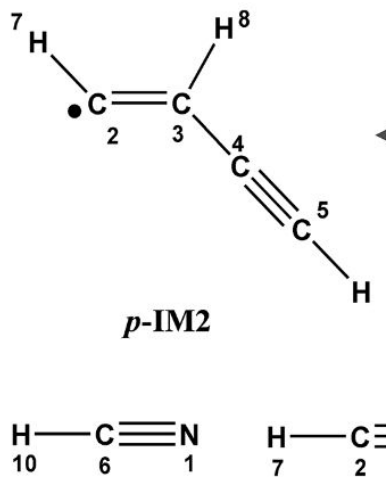

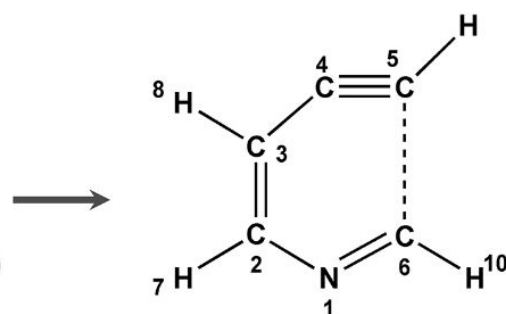

p-TS1
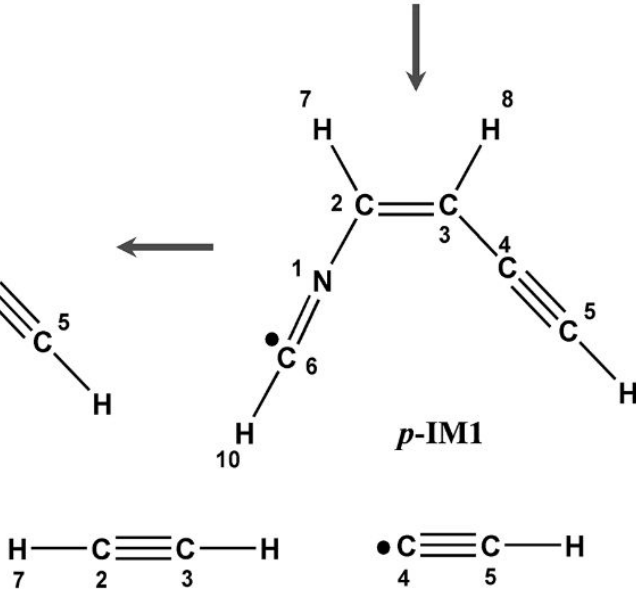

Figure S12. The reaction pathways and products of $\boldsymbol{p}$-pyridyl.
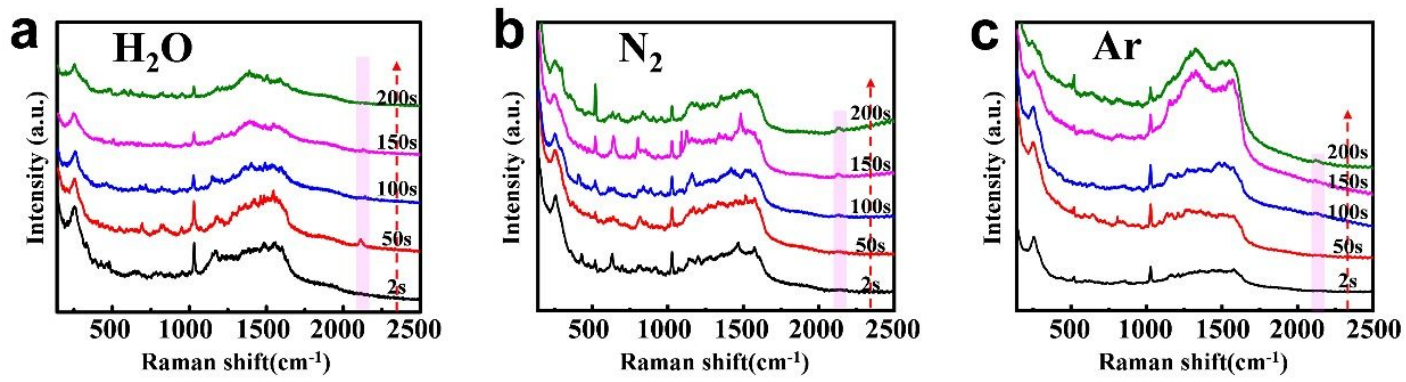

Figure S13. The SERS curves of the sample in different medium. 

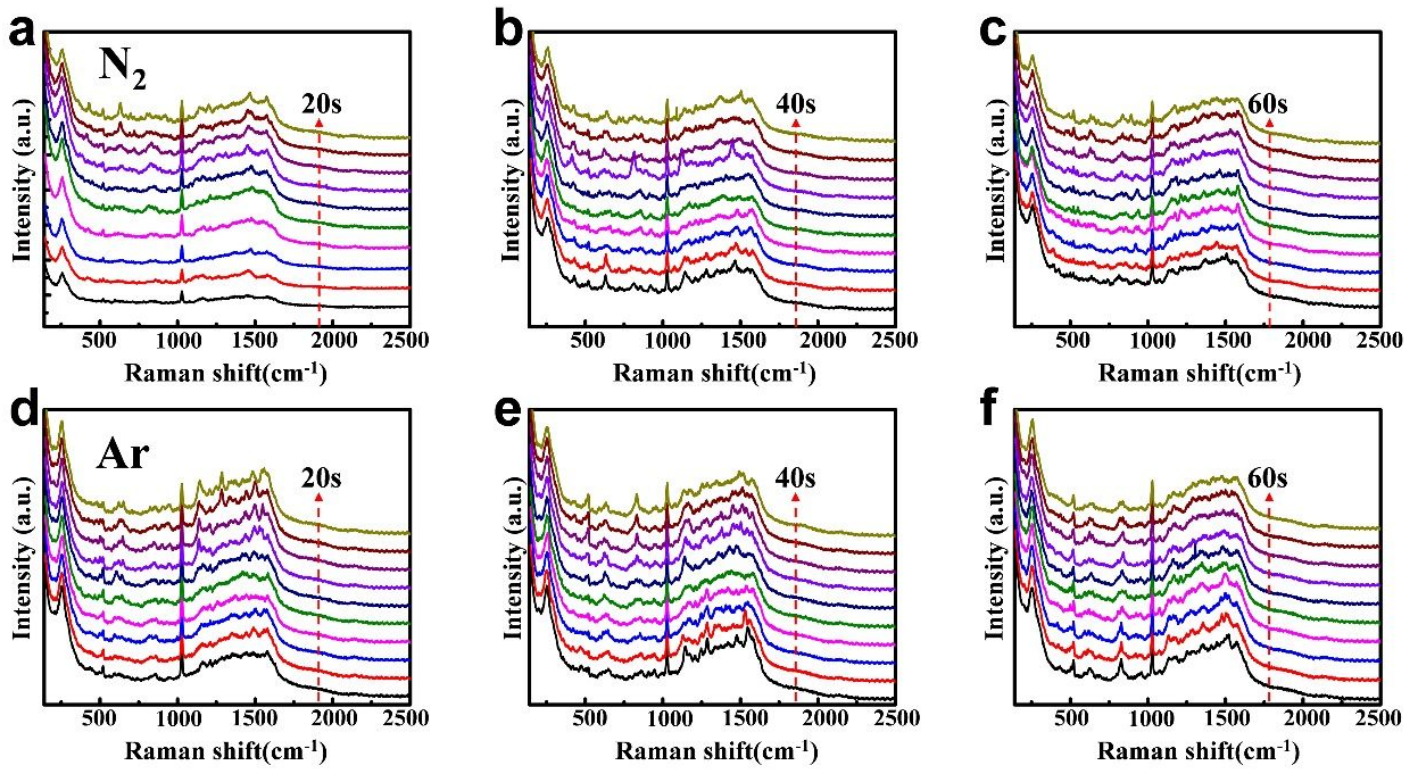

Figure S14. The Raman curves of SRES platform at the first sixty second in the $\mathrm{N}_{2}$ (a-c) and Ar (d-f).
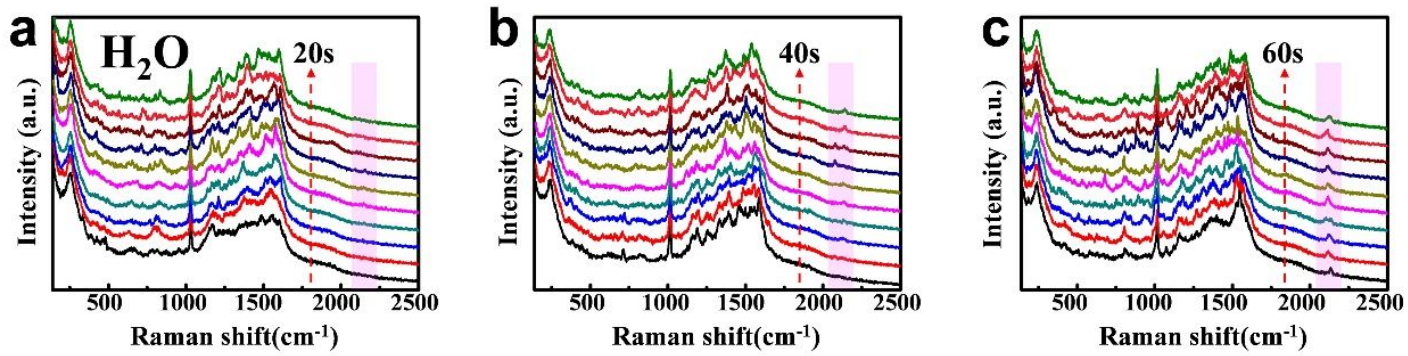

Figure S15. The Raman curves of SRES platform at the first sixty second in the aqueous water (a-c).
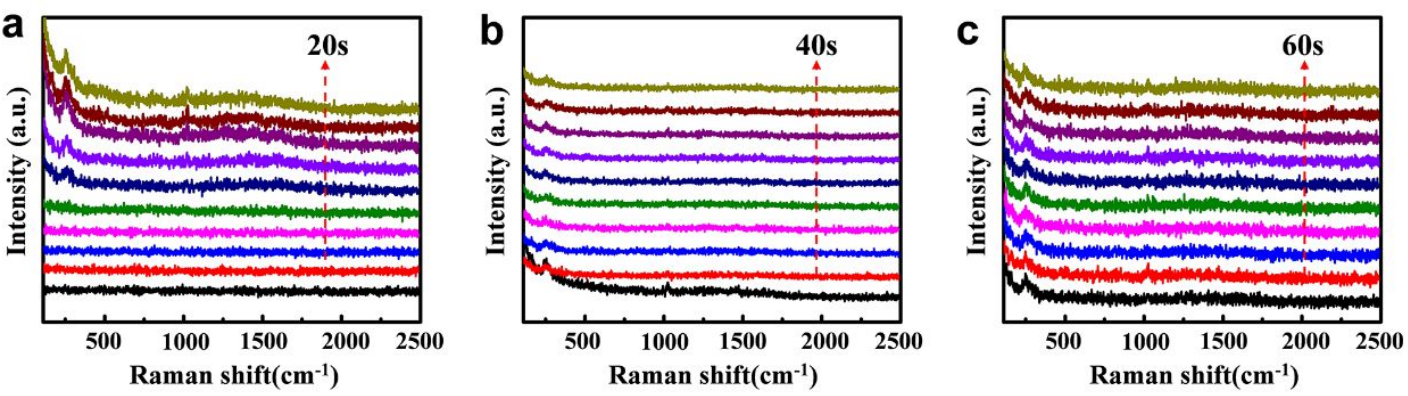

Figure S16. The Raman curves of SRES platform at the first sixty second in the air

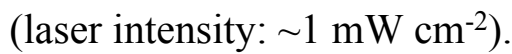



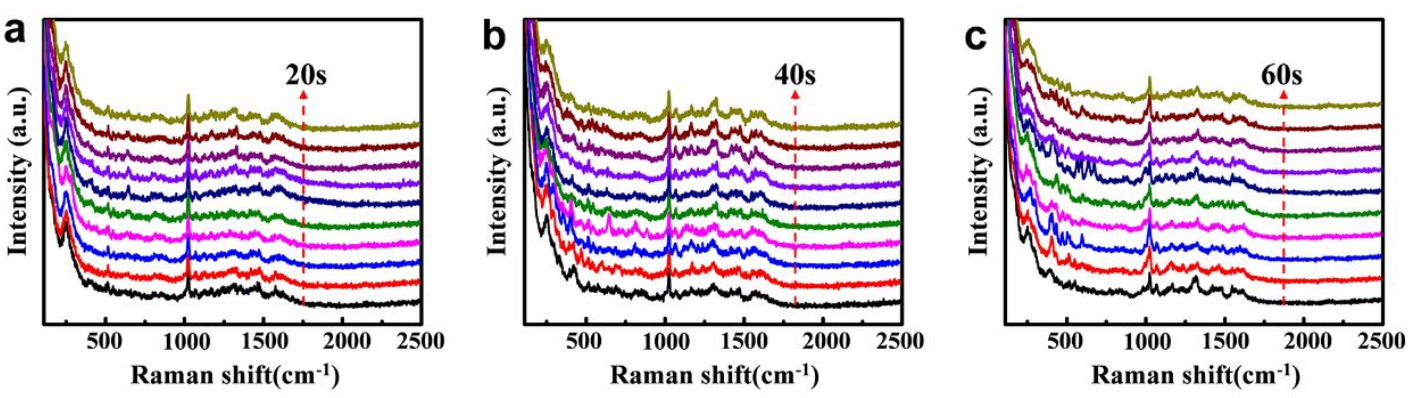

Figure S17. The Raman curves of SRES platform at the first sixty second in the air (laser intensity: $\sim 6 \mathrm{~mW} \mathrm{~cm}^{-2}$ ).
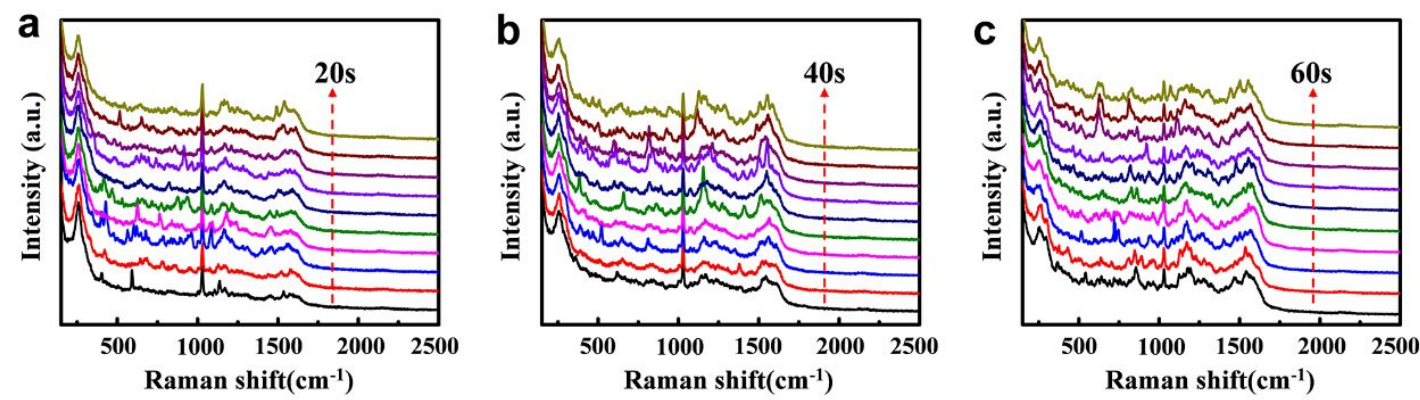

Figure S18. The Raman curves of SRES platform at the first sixty second in the air (laser intensity: $\sim 30 \mathrm{~mW} \mathrm{~cm}^{-2}$ ).
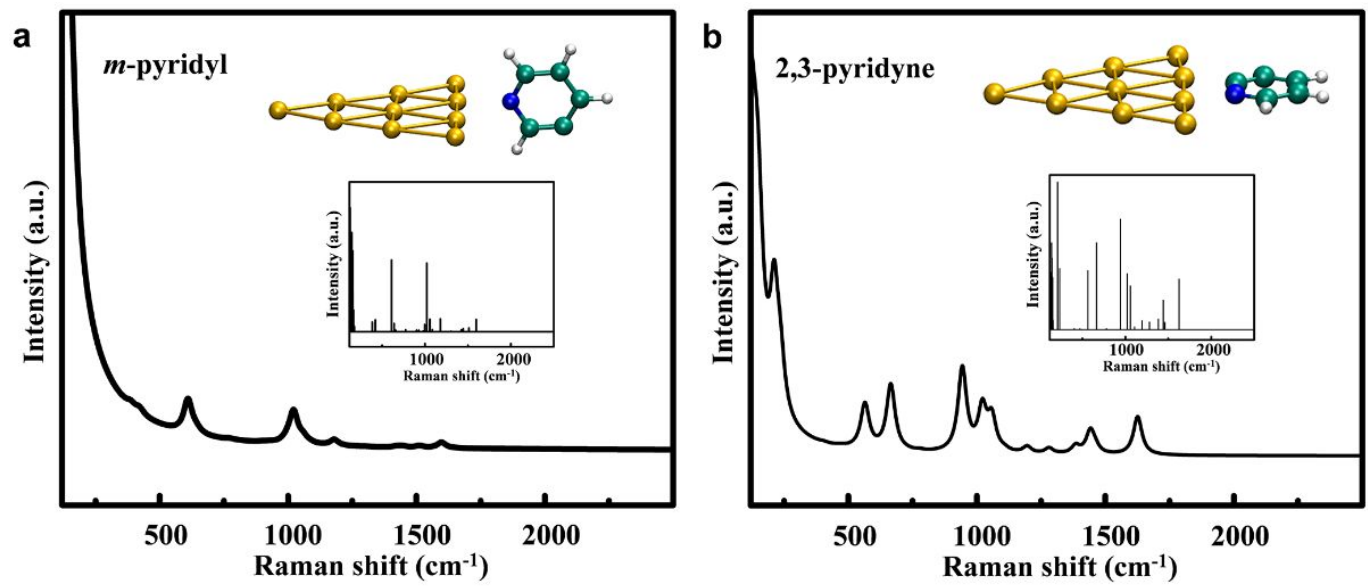

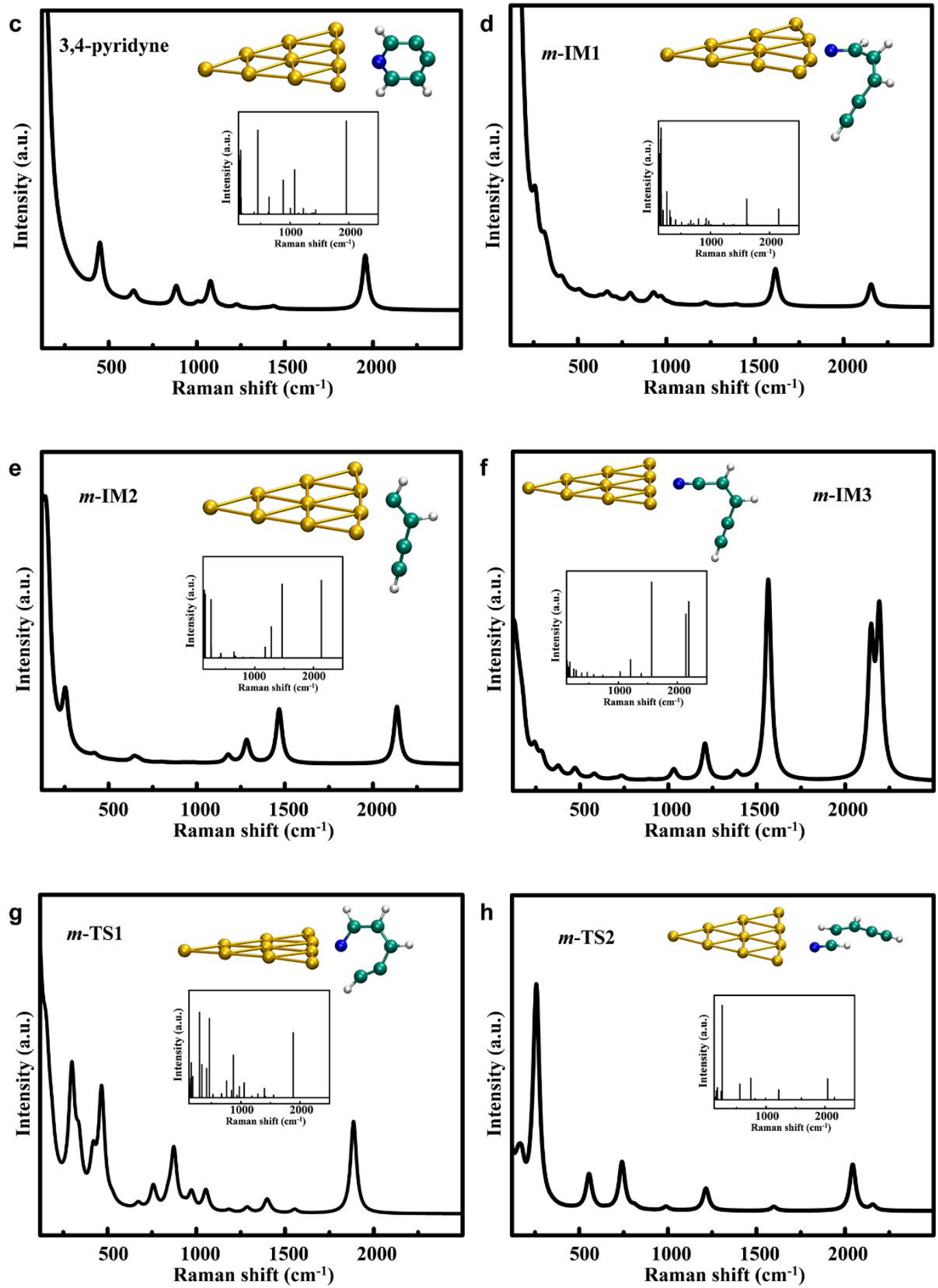

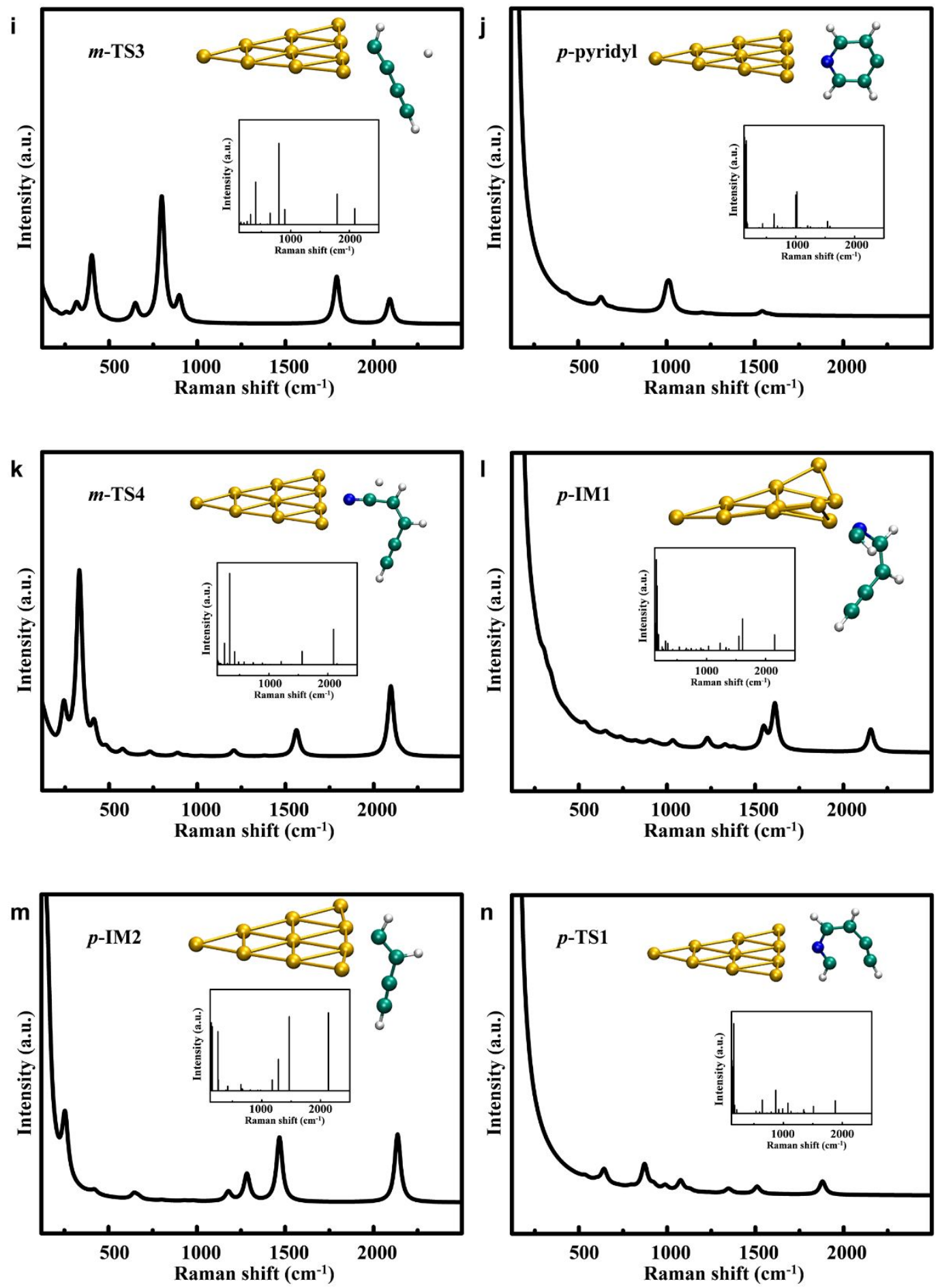


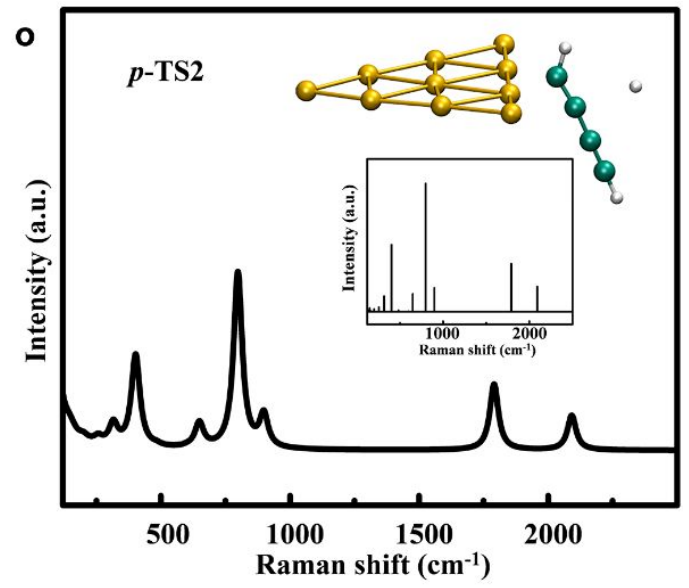

Figure S19. The DFT calculations results of the Au-intermediates.

\section{Reference}

(1) Lu, F.; Zhang, Y.; Liu, S.; Lu, D.; Su, D.; Liu, M.; Zhang, Y.; Liu, P.; Wang, J. X.; Adzic, R. R.; Gang, O. Surface Proton Transfer Promotes Four-Electron Oxygen Reduction on Gold Nanocrystal Surfaces in Alkaline Solution. J. Am. Chem. Soc. 2017, 139, 7310-7317.

(2) Wei, W.; Wang, Y.; Ji, J.; Zuo, S.; Li, W.; Bai, F.; Fan, H. Fabrication of Large-Area Arrays of Vertically Aligned Gold Nanorods. Nano Lett. 2018, 18, 4467-4472.

(3) M.T. Frisch, G. S., H. Scuseria, G. Robb, M. Cheeseman, J. Scalmani, G. Barone, V. Mennucci, B. Petersson, R.E.G.I. et al. Gaussian 09. Wallingford CT 2013.

(4) Humphrey, W.; Dalke, A.; Schulten, K. VMD: Visual molecular dynamics. J. Mol. Graphics 1996, 14, 33-38.

(5) Cheng, X. L.; Zhao, Y. Y.; Zhou, Z. Y. Theoretical studies on p-pyridyl radical decomposition reactions. J MOL STRUC-THEOCHEM 2004, 678, 17-21.

(6) Cheng, X.; Niu, L.; Zhao, Y.; Zhou, Z. Vibrational analysis for multi-channel decomposition reactions of o-pyridyl radical based on DFT methods. Spectrochim. Acta, Pt. A: 
Mol. Biomol. Spectrosc. 2004, 60, 907-914.

(7) Zhan, C.; Wang, Z. Y.; Zhang, X. G.; Chen, X. J.; Huang, Y. F.; Hu, S.; Li, J. F.; Wu, D. Y.; Moskovits, M.; Tian, Z. Q. Interfacial Construction of Plasmonic Nanostructures for the Utilization of the Plasmon-Excited Electrons and Holes. J. Am. Chem. Soc. 2019, 141, 8053-8057.

(8) Cheng, X. Reaction mechanism of decomposition system of m-pyridyl radical: A theoretical investigation. J MOL STRUC-THEOCHEM 2005, 731, 89-99.

(9) Hesse, E.; Creighton, J. A. Investigation by Surface-Enhanced Raman Spectroscopy of the Effect of Oxygen and Hydrogen Plasmas on Adsorbate-Covered Gold and Silver Island Films. Langmuir 1999, 15, 3545-3550.

(10) Murray, C. A.; Bodoff, S. Cyanide adsorption on silver and gold overlayers on island films as determined by surface enhanced Raman scattering. J CHEM PHYS 1986, 85, $573-584$.

(11) Bozzini, B.; Mele, C.; Romanello, V. Time-dependent in situ SERS study of CN adsorbed on gold. J. Electroanal. Chem. 2006, 592, 25-30.

(12) Harvey, C. E.; Weckhuysen, B. M. Surface- and Tip-Enhanced Raman Spectroscopy as Operando Probes for Monitoring and Understanding Heterogeneous Catalysis. Catal. Lett. 2015, 145, 40-57.

(13) von Raben, K. U.; Chang, R. K.; Laube, B. L. Surface enhanced raman scattering of $\mathrm{Au}(\mathrm{CN}) 2-$ ions adsorbed on gold colloids. Chem. Phys. Lett. 1981, 79, 465-469.

(14) Jacobs, M. B.; Jagodzinski, P. W.; Jones, T. E.; Eberhart, M. E. A Surfaced-Enhanced Raman Spectroscopy and Density Functional Theory Study of [Au(CN)2] 
-/[Au(CN)4]- Adsorbed on Gold Nanoparticles. J. Phys. Chem. C2011, 115, 24115-24122.

(15) Premasiri, W. R.; Clarke, R. H.; Londhe, S.; Womble, M. E. Determination of cyanide in waste water by low-resolution surface enhanced Raman spectroscopy on sol-gel substrates. J. Raman Spectrosc. 2001, 32, 919-922.

(16) Liao, P. F.; Stern, M. B. Surface-enhanced Raman scattering on gold and aluminum particle arrays. Opt. Lett. 1982, 7, 483-485.

(17) Senapati, D.; Dasary, S. S. R.; Singh, A. K.; Senapati, T.; Yu, H.; Ray, P. C. A Label-Free Gold-Nanoparticle-Based SERS Assay for Direct Cyanide Detection at the Parts-per-Trillion Level. Chem. Eur. J. 2011, 17, 8445-8451.

(18) Li, R.; Kobayashi, H.; Tong, J.; Yan, X.; Tang, Y.; Zou, S.; Jin, J.; Yi, W.; Fan, J. Radical-Involved Photosynthesis of AuCN Oligomers from Au Nanoparticles and Acetonitrile. J. Am. Chem. Soc. 2012, 134, 18286-18294.

(19) Guttentag, A. I.; Barr, K. K.; Song, T.-B.; Bui, K. V.; Fauman, J. N.; Torres, L. F.; Kes, D. D.; Ciomaga, A.; Gilles, J.; Sullivan, N. F.; Yang, Y.; Allara, D. L.; Zharnikov, M.; Weiss, P. S. Hexagons to Ribbons: Flipping Cyanide on Au\{111\}. J. Am. Chem. Soc. 2016, 138, 15580-15586.

(20) Wang, Y.-H.; Liang, M.-M.; Zhang, Y.-J.; Chen, S.; Radjenovic, P.; Zhang, H.; Yang, Z. L.; Zhou, X. S.; Tian, Z. Q.; Li, J. F. Probing Interfacial Electronic and Catalytic Properties on Well-Defined Surfaces by Using In Situ Raman Spectroscopy. Angew. Chem. Int. Ed. 2018, $57,11257-11261$.

(21) Matz, D. L.; Schalnat, M. C.; Pemberton, J. E. Reaction of Thin Films of Solid-State Benzene and Pyridine with Calcium. J. Am. Chem. Soc. 2012, 134, 12989-12997. 
(22) Wu, D. Y.; Ren, B.; Jiang, Y. X.; Xu, X.; Tian, Z. Q. Density Functional Study and Normal-Mode Analysis of the Bindings and Vibrational Frequency Shifts of the Pyridine-M ( $M$ $=\mathrm{Cu}, \mathrm{Ag}, \mathrm{Au}, \mathrm{Cu}+, \mathrm{Ag}+, \mathrm{Au}+$, and Pt) Complexes. J. Phys. Chem. A. 2002, 106, 9042-9052. 